\title{
SPECTRA, PSEUDOSPECTRA, AND LOCALIZATION FOR RANDOM BIDIAGONAL MATRICES*
}

\author{
LLOYD N. TREFETHEN ${ }^{\dagger}$, MARCO CONTEDINI ${ }^{\ddagger}$, AND MARK EMBREE $§$
}

\begin{abstract}
There has been much recent interest, initiated by work of the physicists Hatano and Nelson, in the eigenvalues of certain random non-hermitian periodic tridiagonal matrices and their bidiagonal limits. These eigenvalues cluster along a "bubble with wings" in the complex plane, and the corresponding eigenvectors are localized in the wings, delocalized in the bubble. Here, in addition to eigenvalues, pseudospectra are analyzed, making it possible to treat the non-periodic analogues of these random matrix problems. Inside the bubble, the resolvent norm grows exponentially with the dimension. Outside, it grows subexponentially in a bounded region that is the spectrum of the infinite-dimensional operator. Localization and delocalization correspond to resolvent matrices whose entries exponentially decrease or increase, respectively, with distance from the diagonal. This article presents theorems that characterize the spectra, pseudospectra, and numerical range for the four cases of finite bidiagonal matrices, infinite bidiagonal matrices ("stochastic Toeplitz operators"), finite periodic matrices, and doubly infinite bidiagonal matrices ("stochastic Laurent operators").
\end{abstract}

Key words. random matrix, Hatano-Nelson problem, spectrum, pseudospectra, Toeplitz matrix, Laurent operator, localization

AMS subject classifications. 15A52, 15A60, 47B35, 47B80

1. Introduction. In this article we investigate random matrices in a class which we denote by

$$
\operatorname{bidiag}_{N}\{X, 1\}
$$

where $X$ is a random variable taking values dense in a compact subset $\operatorname{supp}(X)$ of the complex plane $\mathbf{C}$. The meaning of this notation is that each $A \in \operatorname{bidiag}_{N}\{X, 1\}$ is an $N \times N$ bidiagonal matrix in which the diagonal entries are independent samples from $X$, the superdiagonal entries are equal to 1 , and all other entries are 0 :

$$
A=\left(\begin{array}{ccccc}
x_{1} & 1 & & & \\
& x_{2} & 1 & & \\
& & \ddots & \ddots & \\
& & & x_{N-1} & 1 \\
& & & & x_{N}
\end{array}\right) .
$$

Such matrices have been considered previously under the name of "one way models" by Brézin, Feinberg, and Zee $[7,17,18]$, except with a corner entry $a_{N 1}=1$ included to make the structure periodic and permit nontrivial eigenvalue analysis. They arise as

* This is a preprint of an article published in Communications on Pure and Applied Mathematics, volume 54, pages 595-623 (2001) copyright 2001, John Wiley \& Sons, Inc. This work was supported by the UK Engineering and Physical Sciences Research Council Grant GR/M12414.

† Oxford University Computing Laboratory, Wolfson Bldg., Parks Rd., Oxford OX1 3QD, UK, LNT@comlab.ox.ac.uk

$\ddagger$ Oxford University Computing Laboratory, Wolfson Bldg., Parks Rd., Oxford OX1 3QD, UK, contedini@comlab.ox.ac.uk

$\S$ Oxford University Computing Laboratory, Wolfson Bldg., Parks Rd., Oxford OX1 3QD, UK, embree@comlab.ox.ac.uk 
a limiting case of the nonsymmetric random periodic tridiagonal matrices introduced in 1996 by Hatano and Nelson [26,27].

While our results hold for general compactly supported probability distributions, two choices of $X$ will be of particular interest, which we denote by

$$
X=\{ \pm 1\} \text { and } X=[-2,2] .
$$

The abbreviation $X=\{ \pm 1\}$ denotes the random variable that takes values \pm 1 with equal probability, generating perhaps the simplest nontrivial class of nonsymmetric random matrices:

$$
A=\left(\begin{array}{ccccc} 
\pm 1 & 1 & & & \\
& \pm 1 & 1 & & \\
& & \ddots & \ddots & \\
& & & \pm 1 & 1 \\
& & & & \pm 1
\end{array}\right) .
$$

Feinberg and Zee call this (again with $a_{N 1}=1$ ) the "one way sign model". The abbreviation $X=[-2,2]$ denotes the random variable that takes values in the interval $[-2,2]$ with uniform probability. The matrices this random variable generates, which are equally easy to analyze, capture some of the essential features associated with the Hatano-Nelson model $[26,26]$, including, as we shall discuss in Section 7 , a "delocalization transition."

We offer this article as a contribution to the heretofore nearly empty intersection of two fields: the study of random matrices, and the study of pseudospectra for matrices that are nonnormal (i.e., matrices whose eigenvectors, even if a complete set exists, cannot be taken to be orthogonal). Random matrices, particularly their eigenvalues, have been a familiar topic for about fifty years in physics [37], statistics $[38,59]$, and numerical analysis [15], and more recently in number theory [3,33]. In condensed matter physics, the subject was made famous by Wigner's semicircle law [58] and the phenomenon of Anderson localization (exponential decay of eigenvectors) [1]. These and many other developments in random matrix theory have emphasized hermitian matrices, the setting for classical quantum mechanics, but random nonhermitian problems have been studied too, since the early work of Ginibre [19], by the authors mentioned in the first two paragraphs and by Efetov, Fyodorov, Girko, Goldsheid, Khoruzhenko, Silvestrov, and Sommers, among others.

Pseudospectra and related quantities for nonnormal matrices and operators were first investigated in the 1970s and 1980s [21,35,55] and became a standard tool in the 1990s $[8,48,49,51]$, with applications in fluid mechanics [54], numerical analysis $[29,42,46,47]$, operator theory [2,5], control theory [30], Markov chains [31], differential equations $[9,10,43]$, and integral equations $[40,41]$. In all of these fields it has been found that in cases of pronounced nonnormality, eigenvalues and eigenvectors alone do not always reveal much about the aspects of the behavior of a matrix or operator that matter in applications, including phenomena of stability, convergence, and resonance, and that pseudo-eigenvalues and pseudo-eigenvectors may do better. We shall show that random bidiagonal matrices also follow this pattern. As has been found previously in studies of Toeplitz matrices $[5,6,45]$ and magnetohydrodynamics [4], the consideration of pseudospectra rather than spectra for these matrices sheds light on the exponential (with respect to $N$ ) sensitivity to perturbations of eigenvalues 
and eigenvectors and eliminates troubling discontinuities in the limit $N \rightarrow \infty$. It also allows the delocalization phenomenon to be analyzed without recourse to eigenvalues or eigenvectors and without the need for nonzero corner entries.

Previous works emphasizing the nonnormal aspects of random nonsymmetric matrices include [11] and [56]. An article on pseudospectra of random triangular matrices is in preparation [16], which will make precise some observations illustrated by experiments in [48] and [55]. The significance of pseudospectra for the Hatano-Nelson model, and the limited significance of eigenvalues, eigenvectors, and corner entries for that problem, have been pointed out by the first author since late 1997 in unpublished communications and in presentations at conferences [50].

Our theorems are summarized in Table 1 at the end of the paper.

2. Spectra and pseudospectra. The spectrum of an $N \times N$ matrix $A$, which we denote by $\Lambda(A)$, is its set of eigenvalues, a finite subset of $\mathbf{C}$ consisting of at most $N$ points. For each $z \in \mathbf{C}$, the resolvent of $A$ at $z$ is $(z-A)^{-1}$, if this matrix exists; here $z-A$ is an abbreviation for $z I-A$, where $I$ is the identity. An equivalent definition of $\Lambda(A)$ is that it is the set of numbers $z \in \mathbf{C}$ for which $(z-A)^{-1}$ does not exist (see, e.g., [32] or [44]).

Since the eigenvalues of a bidiagonal matrix are its diagonal entries, the spectrum of any $A \in \operatorname{bidiag}_{N}\{X, 1\}$ is a subset of $\operatorname{supp}(X)$. Thus there is not much to say about the spectra of these matrices. The most interesting fact is that they behave discontinuously in the limit $N \rightarrow \infty$, as can be seen by comparing our Theorems 1 and 2 . Such a discontinuity was pointed out by Davies [11] and Goldsheid and Khoruzhenko [24], and as already noted, it is one motivation for considering pseudospectra instead.

The pseudospectra of a matrix $A$ are compact sets in the complex plane. They depend on vector and matrix norms, and all our discussion is based on the familiar choices

$$
\|x\|=\left(\sum\left|x_{j}\right|^{2}\right)^{1 / 2}, \quad\|A\|=\sup _{\|x\|=1}\|A x\|,
$$

although many of our statements can be generalized to other norms. The 0-pseudospectrum $\Lambda_{0}(A)$ is the same as the spectrum $\Lambda(A)$, and for $\epsilon>0$, the $\epsilon$-pseudospectra are strictly nested sets that expand to fill the plane as $\epsilon \rightarrow \infty$. We can define $\Lambda_{\epsilon}(A)$ in various equivalent ways:

Definition 1. For any $\epsilon \geq 0$, the $\epsilon$-pseudospectrum $\Lambda_{\epsilon}(A)$ of a matrix $A$ is the set of numbers $z \in \mathbf{C}$ satisfying any of the equivalent conditions

(i) $\left\|(z-A)^{-1}\right\| \geq \epsilon^{-1}$;

(ii) $\sigma_{\min }(z-A) \leq \epsilon$;

(iii) $\|A u-z u\| \leq \epsilon$ for some vector $u$ with $\|u\|=1$;

(iv) $z$ is an eigenvalue of $A+E$ for some matrix $E$ with $\|E\| \leq \epsilon$.

Here $\sigma_{\min }$ denotes the smallest singular value, and we follow the convention of writing $\left\|(z-A)^{-1}\right\|=\infty$ if $z \in \Lambda(A)$.

Conditions (i) and (ii) assert that $\Lambda_{\epsilon}(A)$ is the subset of the complex plane bounded by the $\epsilon^{-1}$-level curve(s) of $\left\|(z-A)^{-1}\right\|$ or the $\epsilon$-level curve(s) of $\sigma_{\min }(z-A)$. Condition (iii) concerns the existence of an $\epsilon$-pseudo-eigenvector. Condition (iv) asserts that $\Lambda_{\epsilon}(A)$ is the set of all complex numbers that are in the spectrum of some 

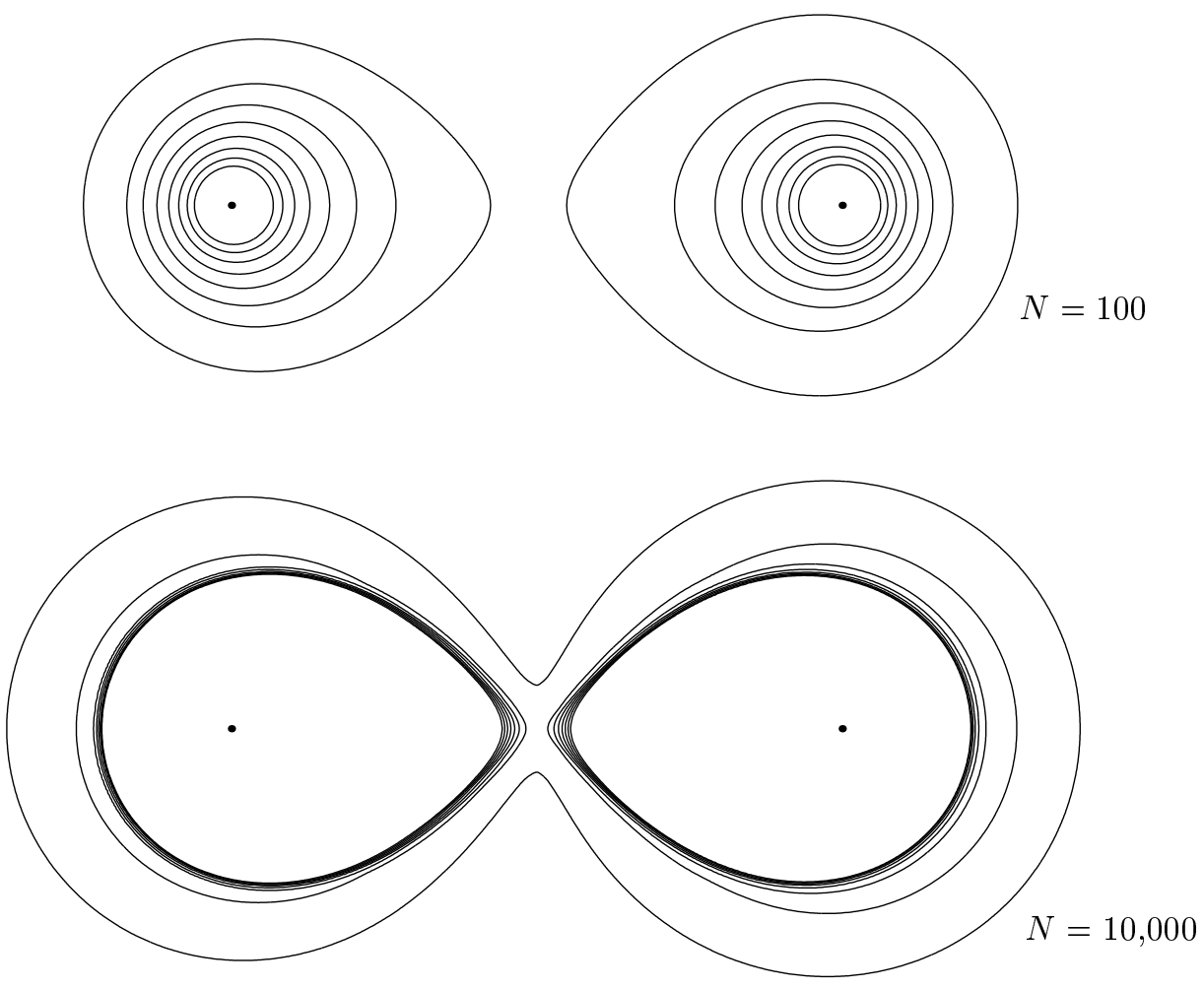

FIG. 1. Spectra and pseudospectra in the complex plane of typical matrices from $\operatorname{bidiag}_{N}\{\{ \pm 1\}, 1\}$. The solid dots, at $z= \pm 1$, are the eigenvalues. The contours, from outside to inside, are the boundaries of $\Lambda_{\epsilon}(A)$ for $\epsilon=10^{-2}, 10^{-6}, 10^{-10}, 10^{-14}, \ldots, 10^{-30}$.

matrix obtained by a perturbation of norm $\leq \epsilon$. The equivalence of (i)-(iv) is discussed in [13], [45], and [47], and much more extensive related material can be found in [32].

Figure 1 shows pseudospectra of two matrices from $\operatorname{bidiag}_{N}\{\{ \pm 1\}, 1\}$, one with $N=100$ and one with $N=10,000 .{ }^{1}$ The pictures reveal that the nonnormality of these matrices is pronounced. The resolvent norm grows exponentially in a region with the form of a figure 8, more precisely a lemniscate, and for large $N$ and small $\epsilon$, the $\epsilon$-pseudospectra have approximately this fixed shape, including points far from

\footnotetext{
${ }^{1}$ In view of condition (ii) of the definition of pseudospectra, pictures like this can be generated by computing $\sigma_{\min }(z-A)$ for values of $z$ on a grid in the complex plane and sending the results to a contour plotter. The work involved is potentially very large, $O\left(N^{3} \nu^{2}\right)$ floating point operations for a $\nu \times \nu$ grid, if the standard $O\left(N^{3}\right)$ dense matrix methods for computing the SVD are used. However, our matrices are bidiagonal, so only "phase 2" of an SVD computation is needed, involving the QR or LR or divide-and-conquer algorithms, and this reduces the operation count to an easily manageable $O\left(N^{2} \nu^{2}\right)$ or $O\left(N \nu^{2}\right)$, depending on whether one computes all the singular values or just the smallest [12]. Since the bidiagonal SVD is not not accessible in MATLAB, we have achieved the speedup to $O\left(N \nu^{2}\right)$ instead by computing $\sigma_{\min }(z-A)$ by Lanczos inverse iteration, as described in [52]. Each plot of Figures 1 and 2 with $N=10,000$ took $5-10$ hours to generate on a Pentium III workstation, essentially the cost of solving on the order of $10^{6}$ bidiagonal systems of $10^{4}$ equations. The plots with $N=100$ each took about ten minutes.
} 

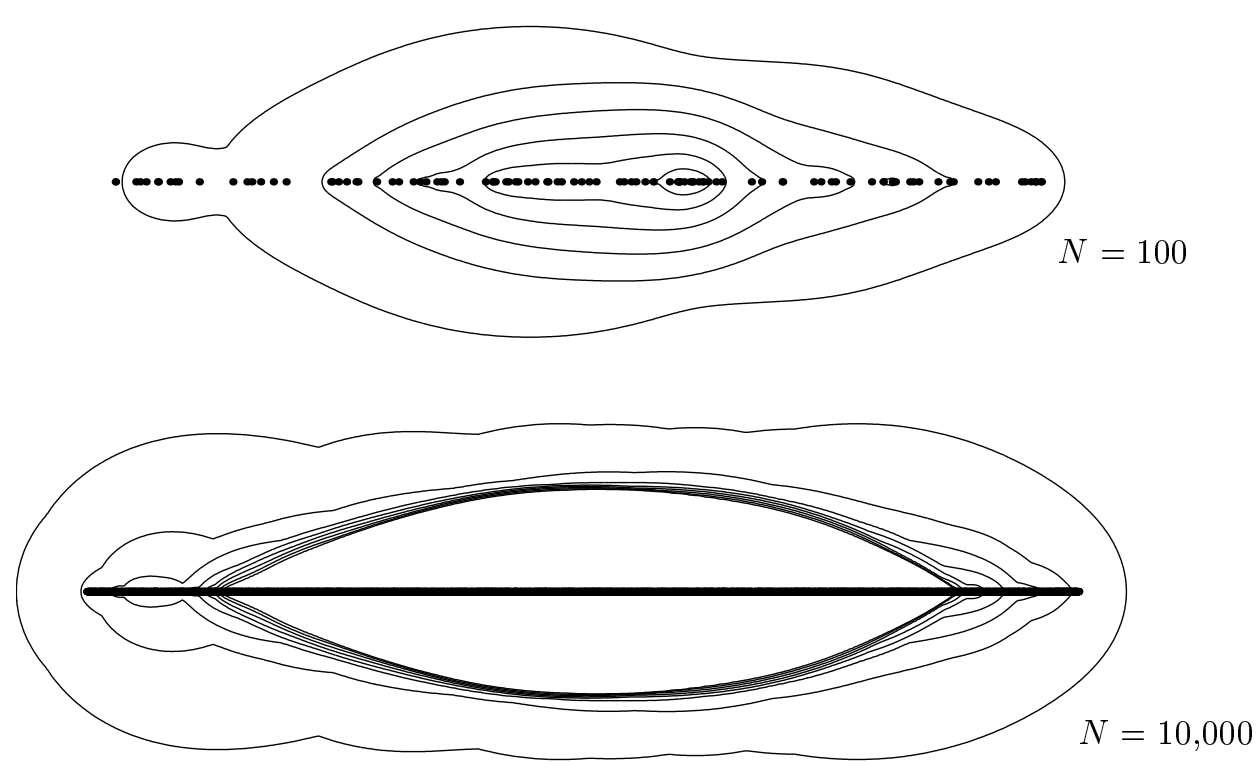

FIG. 2. Same as Figure 1 but for $\operatorname{bidiag}_{N}\{[-2,2], 1\}$. The eigenvalues are now distributed in the interval $[-2,2]$; in the lower plot, they appear to have fused into a solid line. The lowest pseudospectral contour that appears in the upper plot is $\epsilon=10^{-22}$. The others are there too, in principle, but hidden under the dots.

the real axis. (An example in [45] considers the pseudospectra of matrices of this form in which the diagonal entries \pm 1 are not random but strictly alternating.) There is also some weaker growth of the resolvent norm outside the lemniscate.

Figure 2 shows analogous pictures for the $\operatorname{case}^{\operatorname{bidiag}_{N}}\{[-2,2], 1\}$. The behavior is similar, except that instead of a lemniscate we see a "Hatano-Nelson bubble." Outside the bubble, there is again modest growth of the resolvent norms, and inside the bubble, the norms are again exponentially large. We see, for example, that a matrix perturbation of norm as small as $10^{-30}$ may again change the eigenvalues for $N=10,000$ completely, making them lie close to the bubble. Introducing a nonzero corner entry $a_{N 1}$ would be an example of such a perturbation. It is a tenet of the theory of pseudospectra, however, that these sets reveal more than just the motion of eigenvalues under perturbation: they have implications for the behavior of the unperturbed matrix too. For example, the strong transient effects that make streamwise streaky structures ubiquitous in high Reynolds number shear flows are linear but non-normal in origin; they can be explained by pseudospectra but not by spectra $[28,54]$. Some estimates of the behavior of a matrix that can be obtained from a knowledge of its pseudospectra are reviewed in [49].

We will now develop theorems that make the observations of Figures 1 and 2 precise.

3. Four subsets of the complex plane. Our results, which are closely related to those of Goldsheid and Khoruzhenko [24] and Brézin, Feinberg, and Zee [7,17,18], are based on the explicit computation of the resolvent of the bidiagonal matrix $A$. In 
the $3 \times 3$ case, since

$$
\left(\begin{array}{ccc}
a^{-1} & -1 & \\
& b^{-1} & -1 \\
& & c^{-1}
\end{array}\right)\left(\begin{array}{ccc}
a & a b & a b c \\
& b & b c \\
& & c
\end{array}\right)=I,
$$

we see that if $x_{j}$ is the $j$ th diagonal entry of $A$, then

$$
\begin{aligned}
& (z-A)^{-1}=\left(\begin{array}{ccc}
z-x_{1} & -1 & \\
& z-x_{2} & -1 \\
& & z-x_{3}
\end{array}\right)^{-1} \\
& =\left(\begin{array}{ccc}
\left(z-x_{1}\right)^{-1} & \left(z-x_{1}\right)^{-1}\left(z-x_{2}\right)^{-1} & \left(z-x_{1}\right)^{-1}\left(z-x_{2}\right)^{-1}\left(z-x_{3}\right)^{-1} \\
\left(z-x_{2}\right)^{-1} & \left(z-x_{2}\right)^{-1}\left(z-x_{3}\right)^{-1} \\
& \left(z-x_{3}\right)^{-1}
\end{array}\right) .
\end{aligned}
$$

The pattern for general $N$ is clear. The $(i, j)$ entry $r_{i j}$ of $(z-A)^{-1}$ for $j \geq i$ is given by

$$
r_{i j}=\prod_{k=i}^{j}\left(z-x_{k}\right)^{-1}
$$

which implies

$$
\log \left|r_{i j}\right|=-\sum_{k=i}^{j} \log \left|z-x_{k}\right|
$$

If $z=x_{k}$ for one of the terms in the product or sum, the formulas are still valid if we define $\left|r_{i j}\right|$ to be $\infty$.

We now ask what (3.1) and (3.2) imply about the behavior of $\left|r_{i j}\right|$ as $j+1-i \rightarrow \infty$. We can establish a framework for this as follows. For any $i, j$, and $N$ with $1 \leq i, j \leq N$, the quantity $\left|r_{i j}\right|$ is a random variable taking values in $[0, \infty]$ determined by the set of matrices bidiag $_{N}\{X, 1\}$. However, it is easily seen that this random variable depends only on $j+1-i$, not on $i$ or $j$ individually or on $N$. Let us call it simply $R_{j+1-i}$. Now by (3.2), we have

$$
-\log R_{j+1-i}=\log \left|z-X_{1}\right|+\log \left|z-X_{2}\right|+\cdots+\log \left|z-X_{j+1-i}\right|,
$$

a sum of independent identically distributed random variables, all equal to $\log |z-X|$. Since $|z-X|$ is bounded above, the expected value of $\log |z-X|$, defined by

$$
E(\log |z-X|)=\int_{\operatorname{supp}(X)} \log |z-x| d \mu(x),
$$

necessarily exists: it is either finite or $-\infty$. Here $\mu$ is the measure defining the random variable $X$, with $\int_{\operatorname{supp}(X)} d \mu(x)=1$. Accordingly, the number $d_{\text {mean }}(z)$ defined by

$$
d_{\text {mean }}(z)=\exp (E(\log |z-X|))
$$


also exists: it is either positive or 0 . (If $z \notin \operatorname{supp}(X)$, it is positive; if $z \in \operatorname{supp}(X)$ it may be either positive or 0 .) We can interpret $d_{\text {mean }}(z)$ as the mean distance of $z$ to $\operatorname{supp}(X)$ in the sense of a geometric mean weighted by $\mu$. For $X=\{ \pm 1\}, d_{\text {mean }}(z)$ is given by

$$
d_{\text {mean }}(z)=\left|z^{2}-1\right|^{1 / 2},
$$

and for $X=[-2,2]$, an easy integral shows that we have

$$
d_{\text {mean }}(z)=\exp \left(-1+\frac{1}{4} \operatorname{Re}[(z+2) \log (z+2)-(z-2) \log (z-2)]\right) .
$$

Variants of (3.5) arise in numerical analysis whenever one deals with problems of polynomial interpolation in equally spaced points [34,53].

Since $E(\log |z-X|)$ exists, the Law of Large Numbers implies that

$$
\frac{-\log R_{j+1-i}}{j+1-i} \rightarrow E(\log |z-X|)
$$

or equivalently

$$
\left(R_{j+1-i}\right)^{1 / j+1-i} \rightarrow \frac{1}{d_{\text {mean }}(z)}
$$

almost surely as $j+1-i \rightarrow \infty[25] .^{2}$ If $d_{\text {mean }}(z)=0$, the meaning of this statement is that $\left(R_{j+1-i}\right)^{1 / j+1-i} \rightarrow \infty$ a.s. as $N \rightarrow \infty$.

For any $X$ and $z$, let $d_{\min }(z)$ and $d_{\max }(z)$ be defined by

$$
d_{\min }(z)=\min _{x \in \operatorname{supp}(X)}|z-x|, \quad d_{\max }(z)=\max _{x \in \operatorname{supp}(X)}|z-x| .
$$

Our three measures of distance of $z$ to $\operatorname{supp}(X)$ are ordered,

$$
0 \leq d_{\min }(z) \leq d_{\text {mean }}(z) \leq d_{\max }(z)<\infty,
$$

and thus they can serve to divide $[0, \infty)$ in general into four subintervals. Our results will distinguish four subsets of $\mathbf{C}$ defined by the conditions

$$
\begin{aligned}
\Omega_{\mathrm{I}}: & d_{\max }(z)<1, \\
\Omega_{\mathrm{II}}: & d_{\text {mean }}(z)<1 \leq d_{\max }(z), \\
\Omega_{\mathrm{III}}: & d_{\min }(z) \leq 1 \leq d_{\text {mean }}(z), \\
\Omega_{\mathrm{IV}}: & 1<d_{\min }(z) .
\end{aligned}
$$

(The number 1 in these conditions would become $|\sigma|$ if (1.4) contained $\sigma$ instead of 1 above the diagonal.) Either or both of $\Omega_{\mathrm{I}}$ and $\Omega_{\mathrm{II}}$ can be empty, but $\Omega_{\mathrm{III}}$ and $\Omega_{\mathrm{IV}}$ are always nonempty. The sets are disjoint, with

$$
\Omega_{\mathrm{I}} \cup \Omega_{\mathrm{II}} \cup \Omega_{\mathrm{III}} \cup \Omega_{\mathrm{IV}}=\mathbf{C} .
$$

\footnotetext{
${ }^{2}$ A condition holds almost surely, abbreviated a.s., if it holds with probability 1. For example, if $x_{1}, x_{2}, \ldots$ is a sequence of independent samples from the uniform distribution $[-2,2]$, then the numbers $1 / x_{j}$ are almost surely all finite.
} 
Any or all of $\Omega_{\mathrm{I}}, \Omega_{\mathrm{II}}$, and $\Omega_{\mathrm{III}}$ may contain a portion of $\operatorname{supp}(X)$, but $\Omega_{\mathrm{IV}}$ lies at a distance 1 from $\operatorname{supp}(X)$.

We summarize the observations above in a lemma.

Lemma 1. For $z \in \mathbf{C}$,

$$
\left(1 / d_{\max }(z)\right)^{k} \leq R_{k} \leq\left(1 / d_{\min }(z)\right)^{k}
$$

and

$$
\left(R_{k}\right)^{1 / k} \rightarrow \frac{1}{d_{\text {mean }}(z)} \quad \text { a.s. as } k \rightarrow \infty .
$$

Thus for $z$ in $\Omega_{\mathrm{I}}, \Omega_{\mathrm{II}}, \Omega_{\mathrm{III}}$, and $\Omega_{\mathrm{IV}}$ we have guaranteed exponential growth, almost sure exponential growth, almost sure exponential decay, and guaranteed exponential decay of $R_{k}$ as $k \rightarrow \infty$, respectively. In particular, these conclusions do not depend on whether or not $z \in \operatorname{supp}(X)$.

Figure 3 illustrates these sets for three examples of random variables $X$. The second and third of these are our choices $\{ \pm 1\}$ and $[-2,2]$, but $\operatorname{since} \operatorname{supp}(X)$ has diameter $\geq 1$, the set $\Omega_{\mathrm{I}}$ is empty in both of these cases. Our first example accordingly takes $X$ to be the random variable distributed uniformly in the complex disk $\{x \in$ C : $\left.|x| \leq \frac{1}{3}\right\}$.

For some of our assertions we will need a stronger convergence result than in Lemma 1. If we make the additional assumption $z \notin \operatorname{supp}(X)$, then $\log |z-X|$ is bounded below as well as above; this is now a random variable with the best possible behavior, including, for example, existence of the variance and all higher moments. From the existence of the variance we could apply the Central Limit Theorem, for example, to conclude that the distribution of $\left(-\log R_{k} / k-\log \left(d_{\text {mean }}(z)\right)\right) / \sqrt{k}$ converges as $k \rightarrow \infty$ to the normal distribution $N(0, \operatorname{Var}(\log |z-X|))$. What we actually need is a somewhat different statement to the effect that the tails of the distribution for finite $k$ are small:

Lemma 2. Let $z \in \mathbf{C} \backslash \operatorname{supp}(X)$ be fixed. For any $\epsilon>0$,

$$
P\left(\left|\left(R_{k}\right)^{1 / k}-\frac{1}{d_{\text {mean }}(z)}\right|>\epsilon\right) \leq C_{1} e^{-C_{2} \sqrt{k}}
$$

for all $k \geq 1$, for some positive constants $C_{1}$ and $C_{2}$.

Sketch of proof. The condition in question is equivalent (with a change of $\epsilon$ ) to the condition

$$
P\left(\left|-\log R_{k}-k E(\log |z-X|)\right|>\epsilon k\right) \leq C_{1} e^{-C_{2} \sqrt{k}},
$$

which is the same as

$$
P\left(|\log | z-x_{1}|+\cdots+\log | z-x_{k}|-k E(\log |z-X|)|>\epsilon k\right) \leq C_{1} e^{-C_{2} \sqrt{k}} .
$$

Let $F(t)$ be the distribution function for $\log |z-X|-E(\log |z-X|)$, and, following a standard technique in probability theory, let $f(\omega)$ denote the characteristic function of $F[25]$,

$$
f(\omega)=\int_{-\infty}^{\infty} e^{i \omega t} F(d t)
$$




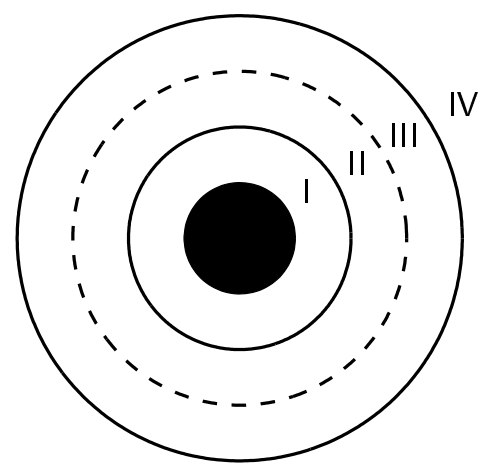

(a) If $X$ is the uniform distribution on $\left\{x \in \mathbf{C}:|x| \leq \frac{1}{3}\right\}$, then $\Omega_{\mathrm{I}}$ is defined by $|z|<2 / 3, \Omega_{\mathrm{II}}$ by $2 / 3 \leq|z|<1, \Omega_{\text {III }}$ by $1 \leq|z| \leq 4 / 3$, and $\Omega_{\text {IV }}$ by $4 / 3<|z|$.

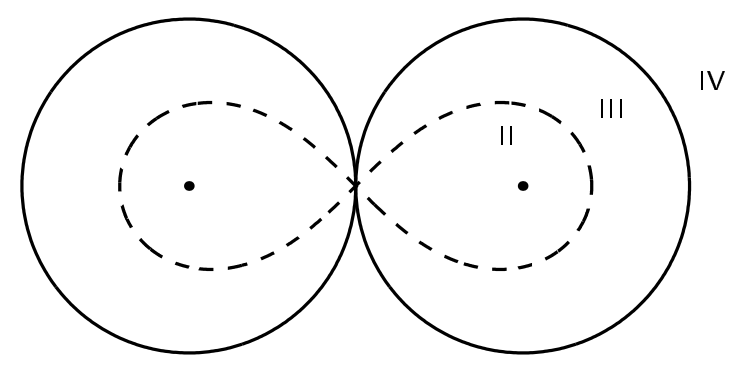

(b) For $X=\{ \pm 1\}, \Omega_{\mathrm{I}}$ is empty, $\Omega_{\mathrm{II}}$ is defined by $\left|z^{2}-1\right|<1, \Omega_{\mathrm{III}}$ by $1 \leq\left|z^{2}-1\right|$ and $\min \{\mid z-$ $1|| z+1 \mid,\} \leq 1$, and $\Omega_{\mathrm{IV}}$ by $\min \{|z-1|,|z+1|\}>1$.

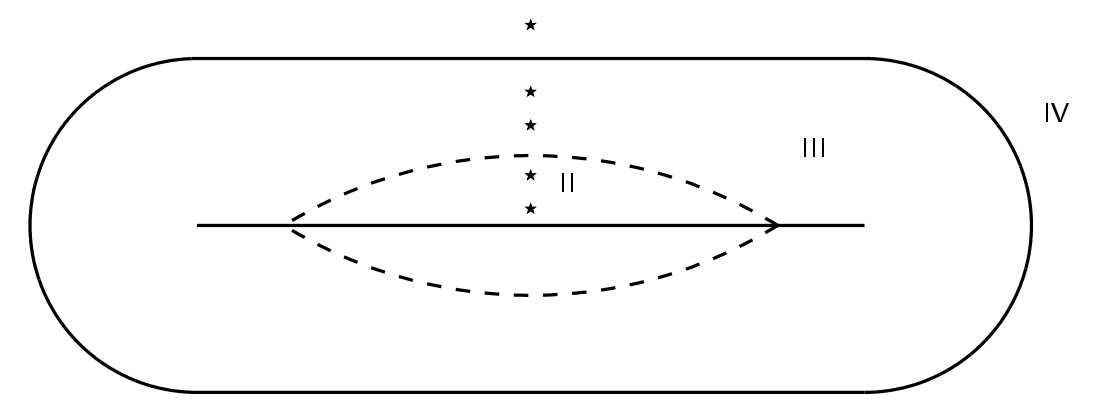

(c) For $X=[-2,2], \Omega_{\mathrm{I}}$ is again empty, $\Omega_{\mathrm{II}}$ is defined by $d_{\text {mean }}(z)<1, \Omega_{\mathrm{III}}$ by $d_{\text {mean }}(z) \geq 1$ and $\min _{x \in[-2,2]}|z-x| \leq 1$, and $\Omega_{\mathrm{IV}}$ by $\min _{x \in[-2,2]}|z-x|>1$, where $d_{\text {mean }}(z)$ is given by $(3 . \overline{5})$. The stars are explained in Figure 4.

FIG. 3. The sets $\Omega_{\mathrm{I}}, \Omega_{\mathrm{II}}, \Omega_{\mathrm{III}}, \Omega_{\mathrm{IV}}$ for three random variables $X$. By Theorem 1 , the resolvent norm of the $N \times N$ matrix grows exponentially as $N \rightarrow \infty$ for $z \in \Omega_{\mathrm{I}}$ (surely) and $\Omega_{\mathrm{II}}$ (almost surely), grows subexponentially in $\Omega_{\mathrm{III}}$ (almost surely), and is bounded independently of $N$ in $\Omega_{\mathrm{IV}}$. The boundary between $\Omega_{\mathrm{II}}$ and $\Omega_{\mathrm{III}}$ (dashed) is the "bubble" of points whose weighted geometric mean distance to $\operatorname{supp}(X)$ is equal to 1 . Theorems $2-4$ relate these regions to the spectra of the corresponding singly infinite, periodic, and doubly infinite matrices. 
which is the Fourier transform of $d F / d t$. The sum of $k$ independent copies of this random variable has the characteristic function $(f(\omega))^{k}$, and the inverse Fourier transform gives

$$
P\left(\left(-\log R_{k}-k E(\log |z-X|)\right)>\epsilon k\right)=\int_{\epsilon k}^{\infty} \frac{1}{2 \pi i} \int_{-\infty}^{\infty} e^{-i \omega t}(f(\omega))^{k} d \omega d t
$$

with a similar integral for values $<-\epsilon$. Now since $X$ has compact support, by the standard theory of characteristic functions, $f(0)=1, f^{\prime}(0)=0,|f(\omega)|<1$ for $\omega \in \mathbf{R} \backslash\{0\}$, and $f^{\prime \prime}(0)<0$ (unless the distribution $\log |z-X|$ has zero variance, in which case the lemma is trivial). Moreover, $f(\omega)$ is an entire function of $\omega$, i.e., analytic throughout the complex $\omega$-plane. This means that the contour of integration of the inner integral in (3.12) can be deformed into the complex plane without changing the value of the integral. In particular, let the real axis be replaced by the complex path $-\infty \rightarrow \alpha \rightarrow \beta \rightarrow \gamma \rightarrow \delta \rightarrow+\infty$, where $\alpha=-k^{-1 / 4}, \beta=-k^{-1 / 4}-i k^{-1 / 2}$, $\gamma=k^{-1 / 4}-i k^{-1 / 2}$, and $\delta=k^{-1 / 4}$. (Instead of the exponent $-1 / 4$ we could take any number in $[-1 / 4,0)$.) On the segment $[\beta, \gamma]$ of this new contour of integration, $\left|e^{-i \omega t}\right| \leq e^{-\epsilon \sqrt{k}}$ since $t \geq \epsilon k$. On the segments $[\alpha, \beta]$ and $[\gamma, \delta],|f(\omega)| \leq 1-(1+$ $o(1))\left|f^{\prime \prime}(0)\right| / 2 \sqrt{k}$, implying $\left|(f(\omega))^{k}\right| \leq(1+o(1)) e^{-\left|f^{\prime \prime}(0)\right| \sqrt{k} / 2}$. On the segments $(-\infty, \alpha]$ and $[\delta, \infty),|f(\omega)|$ is bounded below 1 and thus $\left|(f(\omega))^{k}\right|$ is exponentially small. Putting together these estimates, we see that the the integrand is bounded over the whole contour by a function of the form $C_{1} e^{-C_{2} \sqrt{k}}$.

4. Random bidiagonal matrices. Lemmas 1 and 2 concerned the entries $\left|r_{i j}\right|$ of the resolvent $(z-A)^{-1}$, but our main interest is in the resolvent norm, which defines the pseudospectra. The following theorem records a number of facts about these norms. It also makes some statements about spectra, which depend on a definition of what it means for a sequence of sets to converge to a limit. For our purposes the following quite strong definition is suitable.

Definition 2. Let $\left\{K_{N}\right\}, N=1,2, \ldots$ be a sequence of subsets of $\mathbf{C}$ and let $K$ be a closed subset of $\mathbf{C}$. We say that

$$
K_{N} \rightarrow K \quad \text { as } N \rightarrow \infty
$$

if for every $\epsilon>0$, there exists an integer $N_{0}$ such that for all $N \geq N_{0}, K_{N}$ and $S$ are each contained in the $\epsilon$-neighborhood of the other. We say that

$$
K_{N} \rightarrow K \quad \text { a.s. as } N \rightarrow \infty
$$

if this condition holds with probability 1.

Theorem 1 (random bidiagonal matrices). Let $A$ be an $N \times N$ matrix of the form (1.2), and let $\Omega_{\mathrm{I}}, \Omega_{\mathrm{II}}, \Omega_{\mathrm{III}}, \Omega_{\mathrm{IV}}, d_{\min }(z), d_{\text {mean }}(z)$, and $d_{\max }(z)$ be defined as in the previous section. Then for $z \in \mathbf{C}$, we have the following conditions on the norm of the resolvent. As always, we write $\left\|(z-A)^{-1}\right\|=\infty$ if $(z-A)^{-1}$ does not exist.

(I) If $z \in \Omega_{\mathrm{I}}$, then $\left\|(z-A)^{-1}\right\| \geq\left(1 / d_{\max }(z)\right)^{N}$ (guaranteed exponential growth).

(II) If $z \in \Omega_{\mathrm{I}} \cup \Omega_{\mathrm{II}}$, then $\left\|(z-A)^{-1}\right\| \rightarrow \infty$ a.s., and if in addition $z \notin \operatorname{supp}(X)$, then $\left\|(z-A)^{-1}\right\|^{1 / N} \rightarrow 1 / d_{\text {mean }}(z)$ a.s. as $N \rightarrow \infty$ (almost sure exponential growth).

(III) If $z \in \Omega_{\mathrm{III}}$, then $\left\|(z-A)^{-1}\right\| \rightarrow \infty$ a.s., and if in addition $z \notin \operatorname{supp}(X)$, then $\left\|(z-A)^{-1}\right\|^{1 / N} \rightarrow 1$ a.s. as $N \rightarrow \infty$ (almost sure subexponential growth). 
(IV) If $z \in \Omega_{\mathrm{IV}}$, then $\left\|(z-A)^{-1}\right\|<1 /\left(d_{\min }(z)-1\right)$ (guaranteed boundedness).

The spectrum satisfies $\Lambda(A) \subseteq \operatorname{supp}(X)$, with $\Lambda(A) \rightarrow \operatorname{supp}(X)$ a.s. as $N \rightarrow \infty$ in the sense of Definition 2. The numerical range satisfies $W(A) \subseteq \operatorname{conv}\left(\Omega_{\mathrm{III}}\right)$, with $W(A) \rightarrow \operatorname{conv}\left(\Omega_{\mathrm{III}}\right)$ a.s. as $N \rightarrow \infty$, where $\operatorname{conv}(\cdot)$ denotes the convex hull.

Proof. Since the norm of a matrix is at least as large as the absolute value of any of its entries, condition (I) follows from Lemma 1 by considering the corner entry $r_{1 N}$ of $(z-A)^{-1}$.

For condition (II), by Lemma 1, consideration of $r_{1 N}$ again is enough to ensure that $\left\|(z-A)^{-1}\right\|^{1 / N}$ is at least as large as $1 / d_{\text {mean }}(z)$ a.s. as $N \rightarrow \infty$ : the precise statement is $\lim \inf _{N \rightarrow \infty}\left\|(z-A)^{-1}\right\|^{1 / N} \geq 1 / d_{\text {mean }}(z)$ almost surely. To complete the proof we must show that $\left\|(z-A)^{-1}\right\|^{1 / N}$ cannot be essentially larger than this, i.e., $\lim \sup _{N \rightarrow \infty}\left\|(z-A)^{-1}\right\|^{1 / N} \leq 1 / d_{\text {mean }}(z)$ almost surely. To do this, we consider the entries $r_{i j}$ of $(z-A)^{-1}$ in two classes: those with $j+1-i \leq \sqrt{N}$ and those with $j+1-i>\sqrt{N}$. By (4.7), the entries in the former class satisfy $\left|r_{i j}\right| \leq\left(1 / d_{\min }(z)\right)^{\sqrt{N}}$ and therefore $\lim \sup _{N \rightarrow \infty} \max _{i, j}\left|r_{i j}\right|^{1 / N} \leq 1$. By Lemma 2, for any $\epsilon>0$, each of the entries in the latter class satisfy $\left|r_{i j}\right|^{1 / \bar{N}}>1 / d_{\text {mean }}(z)+\epsilon$ with a probability that decreases as $N \rightarrow \infty$ faster than the inverse of any polynomial in $N$. Since there are only polynomially many entries in the matrix, it follows that the same statement about probabilities is true of $\max _{i j}\left|r_{i j}\right|^{1 / N}$ and of $\left\|(z-A)^{-1}\right\|^{1 / N}$. Since $\epsilon$ is arbitrary, it follows that $\lim \sup _{N \rightarrow \infty}\left\|(z-A)^{-1}\right\|^{1 / N} \leq 1 / d_{\text {mean }}(z)$ almost surely.

For condition (IV), we note that $\left\|(z-A)^{-1}\right\| \leq\left\|D_{0}\right\|+\left\|D_{1}\right\|+\cdots+\left\|D_{N-1}\right\|$, where $D_{j}$ denotes the $j$ th upper diagonal of $(z-A)^{-1}$. Since $\left\|D_{j}\right\| \leq d_{\min }(z)^{-1-j}$ by Lemma 1 , the result follows on summing a finite geometric series.

This leaves condition (III) of the resolvent norm estimates, which is the most interesting one, since the resolvent norms are diverging to $\infty$ while the corner entries $r_{1 N}$ are converging to zero. What is going on here is that although on average the entries of the resolvent decrease away from the diagonal, arbitrarily large pockets must (almost surely) appear in which they increase, a phenomenon we shall consider from another angle in Section 6 . To be precise, for $z \in \Omega_{\mathrm{III}}$, since $d_{\min }(z) \leq 1$, there exists $x_{0} \in \operatorname{supp}(X)$ with $\left|z-x_{0}\right| \leq 1$. For any $\epsilon>0$, there is a positive probability that a random sample $x \in X$ will satisfy $\left|x-x_{0}\right|<\epsilon$. It follows that for any $J>0$, a sequence of matrices $A_{1}, A_{2}, \ldots$ from $\operatorname{bidiag}_{N}\{X, 1\}$ for $N=1,2, \ldots$ almost surely eventually contains a block with diagonal entries $x_{j}$, of length at least $J$, with $\left|x_{j}-x_{0}\right|<\epsilon$. The resolvent $(z-A)^{-1}$ of such a matrix contains a $J \times J$ square block along the diagonal determined by these $J$ entries, and by taking $\epsilon$ sufficiently small and $J$ sufficiently large, we can make the norm of this square block arbitrarily large. The proof of $\left\|(z-A)^{-1}\right\| \rightarrow \infty$ a.s. is finished by noting that the norm of the square block is a lower bound for $\left\|(z-A)^{-1}\right\|$. To prove that $\left\|(z-A)^{-1}\right\|^{1 / N} \rightarrow 1$ a.s., on the other hand, we can reason as in the second part of (II), above. As in that argument, the entries with $j+1-i \leq \sqrt{N}$ have $N$ th roots bounded by 1 in the limit $N \rightarrow \infty$, because of the absolute bound on $\left|r_{i j}\right|$, and the entries with $j+1-i>\sqrt{N}$ almost surely have the same property because of Lemma 2 .

Concerning the spectrum, the inclusion $\Lambda(A) \subseteq \operatorname{supp}(X)$ is trivial. To establish that $\Lambda(A) \rightarrow \operatorname{supp}(X)$ a.s. as $N \rightarrow \infty$ we must show in addition that for any $\epsilon$, with probability 1 , there exists an integer $N_{0}$ such that for all $N \geq N_{0}, \operatorname{supp}(X)$ is covered by the $\epsilon$-neighborhood of $\Lambda\left(A_{N}\right)$. Such a result holds pointwise for each $z \in \operatorname{supp}(X)$ individually, and the extension to $\operatorname{supp}(X)$ as a set is an easy matter of compactness.

Concerning the numerical range, the inclusion $W(A) \subseteq \operatorname{conv}\left(\Omega_{\mathrm{III}}\right)$ follows from the inequality $\left\|(z-A)^{-1}\right\| \leq 1 /\left(d_{\min }(z)-1\right)$ for all $z \in \Omega_{\mathrm{IV}}$, thus including all 
$z \notin \operatorname{conv}\left(\Omega_{\mathrm{III}}\right)$. The limit $W(A) \rightarrow \operatorname{conv}\left(\Omega_{\mathrm{III}}\right)$ a.s. follows from this together with the fact that $\left\|(z-A)^{-1}\right\| \rightarrow \infty$ a.s. for $z \in \Omega_{\mathrm{III}}$.

Theorem 1 explains the results of Figures 1 and 2. In particular, it explains the exponential growth of the resolvent norms inside the bubble and the slower growth outside. We see that the bubble is defined by the condition $d_{\text {mean }}(z)=1$, i.e., it is the set of points whose weighted geometric mean distance to $\operatorname{supp}(X)$ is equal to 1 ; we shall make more of this definition in Section 7. Feinberg and Zee [17] obtained the same formula for the location of eigenvalues in their periodic one way model, based on a similar result by Goldsheid and Khoruzhenko [24] for the eigenvalues of the Hatano-Nelson model.

A more quantitative confirmation of Theorem 1 is presented in Figure 4. Here, for a succession of randomly selected matrices $A \in \operatorname{bidiag}_{N}\{[-2,2], 1\}$ for various dimensions $N$, the norm $\left\|(z-A)^{-1}\right\|$ is plotted for the five values of $z$ in $\Omega_{\mathrm{II}}, \Omega_{\mathrm{III}}$, and $\Omega_{\mathrm{IV}}$ marked by stars in Figure $3(\mathrm{c})$. As predicted, we see exponential growth in the first case, subexponential growth in the second, and no growth in the third.

Figure 5 shows numerically computed boundaries of the $\epsilon$-pseudospectra of a single matrix $A \in \operatorname{bidiag}_{N}\{[-2,2], 1\}$ of dimension $N=10^{6}$ for $\epsilon=10^{-1}, 10^{-2}$, and $10^{-100}$. Note that the $10^{-100}$-pseudospectrum is almost identical to the region inside the "bubble", plus two "wings" (actually strings of very small connected components around the eigenvalues) extending outside. Inside this region we have $\left\|(z-A)^{-1}\right\|>$ $10^{100}$, and the norms at the points $0.3 i$ and $0.1 i$ indicated by stars in Figure 3 are approximately $10^{35804}$ and $10^{99698}$, respectively. In an application governed by this operator one would expect that for practical purposes, the behavior would be as if the spectrum included all of the region inside the bubble.

We do not know of a previously published plot of a $10^{-100}$-pseudospectrum, or of pseudospectra of a matrix with dimension in the millions.

5. Random bidiagonal infinite matrices. For an operator acting on a space of infinite dimension, complications arise in spectral theory that are absent for matrices. It is no longer the case that the spectrum is just the set of eigenvalues. Instead, for a bounded operator $A$ acting in Hilbert space (which is the natural context of our problem, though various generalizations are possible), $\Lambda(A)$ is the set of numbers $z \in \mathbf{C}$ for which $z-A$ does not have a bounded inverse $(z-A)^{-1}[20,32,44]$.

Let us be specific about our bidiagonal matrices $A$ of the form (1.2). In the infinite dimensional limit, we could consider a singly infinite bidiagonal matrix acting on vectors $\left(v_{1}, v_{2}, \ldots\right)^{T}$. We like to think of such an $A$ as a bidiagonal stochastic Toeplitz operator, with domain $\ell_{2}^{+}=\ell_{2}\left(\mathbf{Z}^{+}\right.$). (A Toeplitz matrix has constant entries on each diagonal; a "stochastic Toeplitz matrix" has independent samples from a fixed distribution on each diagonal.) Alternatively, we could consider a doubly infinite bidiagonal matrix acting on vectors $\left(\ldots, v_{-1}, v_{0}, v_{1}, \ldots\right)^{T}$. We think of this as a bidiagonal stochastic Laurent operator, with domain $\ell_{2}=\ell_{2}(\mathbf{Z})$.

Roughly speaking, for these stochastic problems as for their non-stochastic Toeplitz and Laurent cousins, the Toeplitz operator corresponds to a limit of Toeplitz matrices and the Laurent operator corresponds to a limit of circulant matrices (i.e., Toeplitz matrices with corner entries introduced to make the structure periodic) as $N \rightarrow \infty$. We can explain this for our bidiagonal matrices as follows. If a singly infinite triangular matrix $B$ has an inverse $B^{-1}$, then it is easily shown by induction that the finite sections of $B^{-1}$ must be the inverses of the corresponding finite sections of $B$. Thus a nonsingular upper triangular Toeplitz operator has an upper triangular 


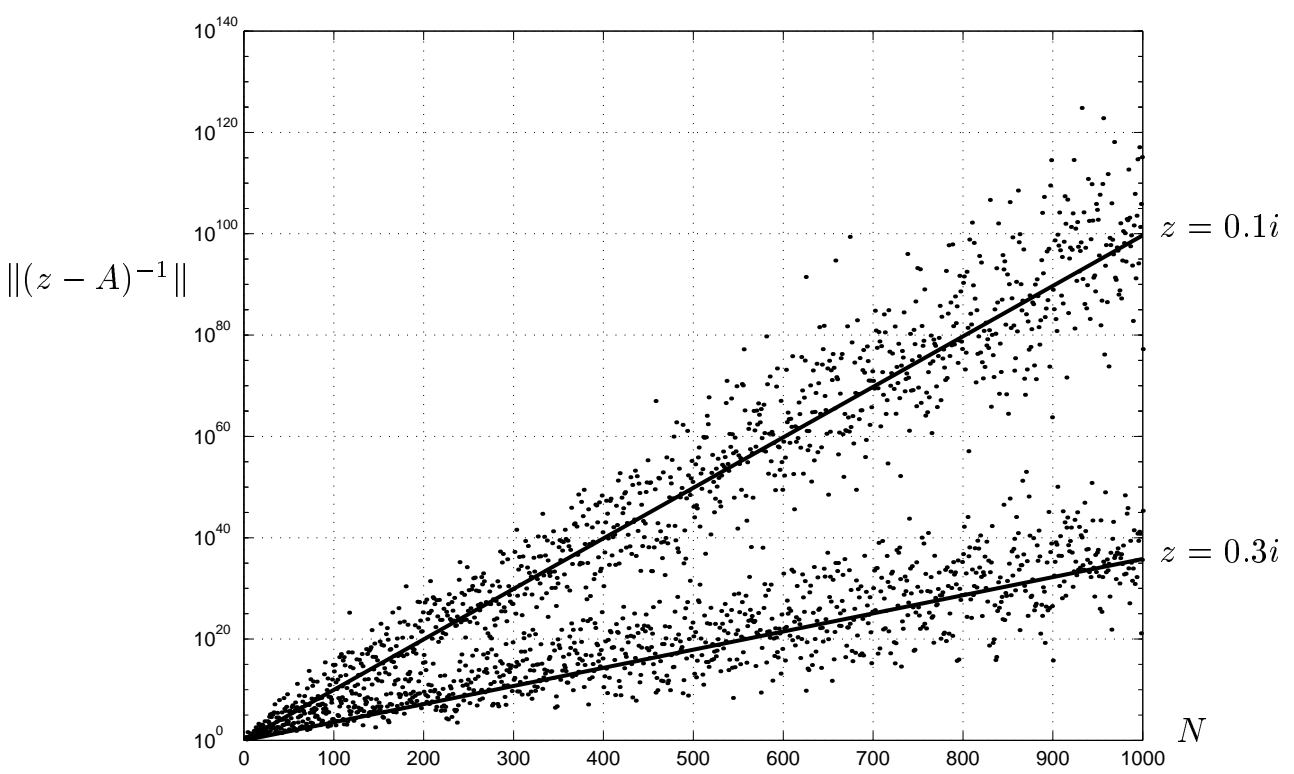

(a) Resolvent norms for two choices $z \in \Omega_{\mathrm{II}}$. The straight lines mark the asymptotic results of Theorem 1 (II).

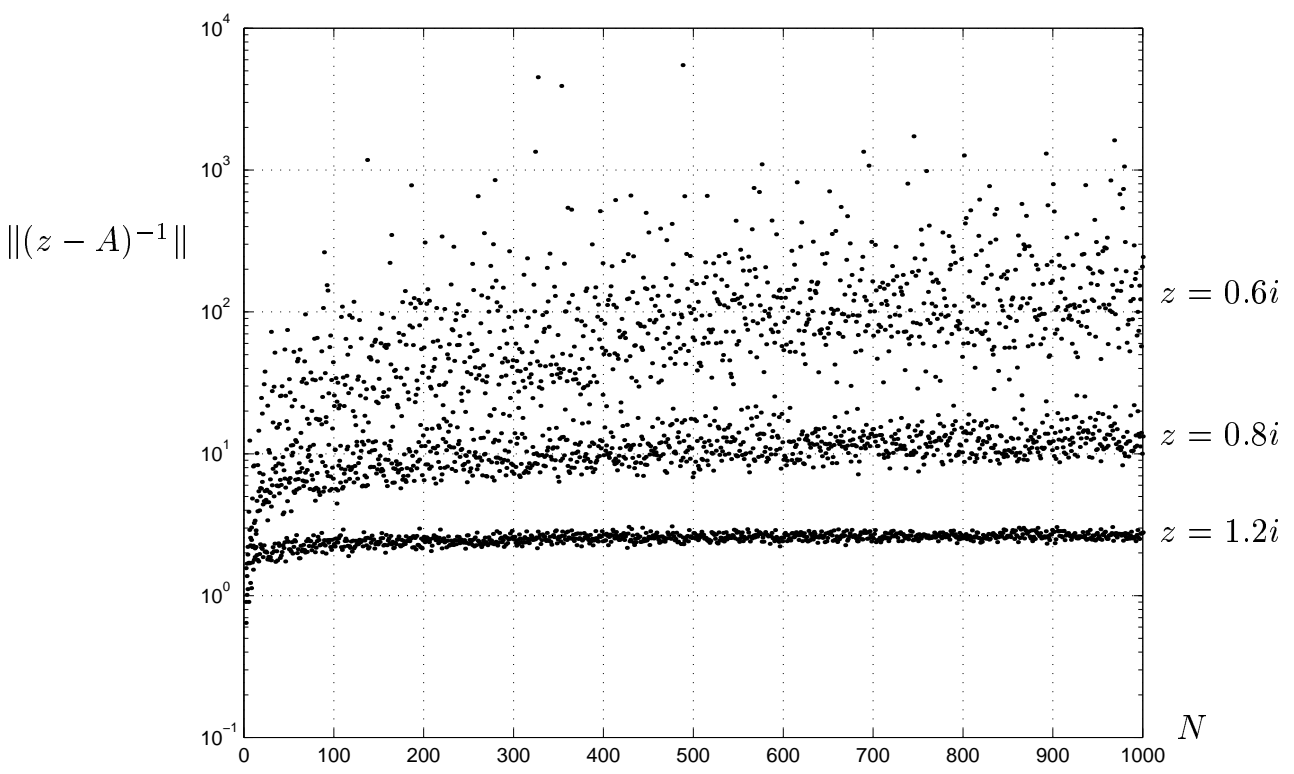

(b) Resolvent norms for two choices $z \in \Omega_{\mathrm{III}}$ and one choice $z \in \Omega_{\mathrm{IV}}$. As guaranteed by Theorem 1 (IV), the latter data are all bounded by $1 /\left(d_{\min }(1.2 i)-1\right)=5$.

FIG. 4. Norms $\left\|(z-A)^{-1}\right\|$ for randomly selected matrices from $\operatorname{bidiag}_{N}\{[-2,2], 1\}$ for $N=$ $2,3, \ldots, 1000$. The five sets of data correspond to the five values of $z$ indicated by stars in Figure 3 . Note the different vertical scales. 


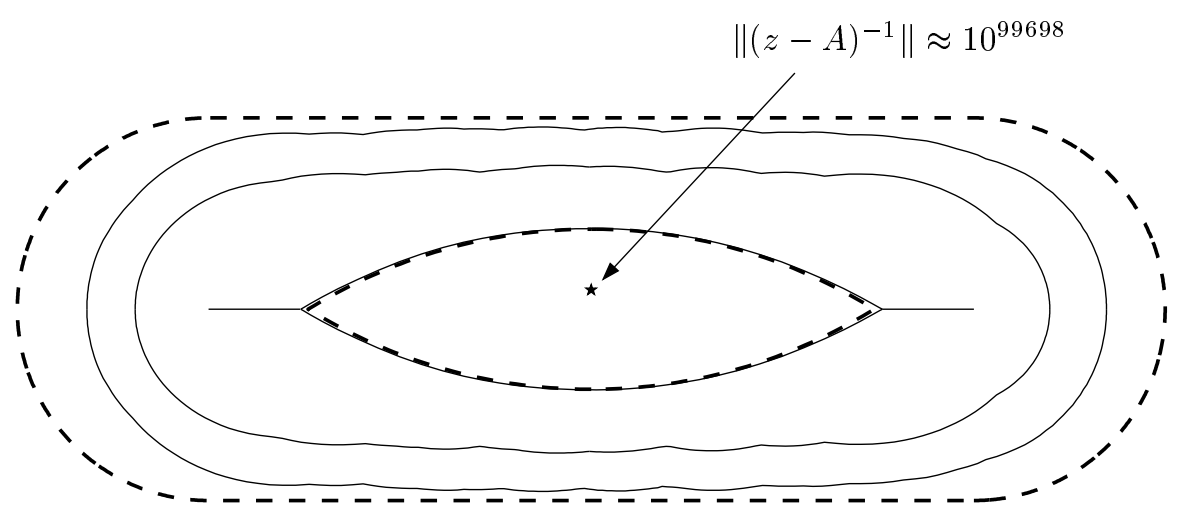

FIG. 5. Numerically computed $\epsilon$-pseudospectra for a single matrix $A \in \operatorname{bidiag}_{N}\{[-2,2], 1\}$ with $N=10^{6}$ for $\epsilon=10^{-1}, 10^{-2}$, and $10^{-100}$. The inner dashed curve is the bubble separating $\Omega_{\mathrm{II}}$ and $\Omega_{\mathrm{III}}$, and the outer dashed curve is the boundary of $\Omega_{\mathrm{III}}$ and $\Omega_{\mathrm{IV}}$. Inside the bubble, the resolvent norm is very large.

inverse, namely the matrix one gets by computing out from the diagonal in the obvious fashion.

Here are results for the singly infinite case; the doubly infinite case is addressed in Section 7.

THEOREM 2 (random bidiagonal infinite matrices). Let $A$ be a singly infinite matrix ("stochastic Toeplitz operator") of the form (1.2). Then $\Omega_{\mathrm{I}} \subseteq \Lambda(A) \subseteq \Omega_{\mathrm{I}} \cup$ $\Omega_{\mathrm{II}} \cup \Omega_{\mathrm{III}}$, and with probability 1 ,

$$
\Lambda(A)=\Omega_{\mathrm{I}} \cup \Omega_{\mathrm{II}} \cup \Omega_{\mathrm{III}} .
$$

For $z \in \Omega_{\mathrm{IV}}$, we have

$$
\left\|(z-A)^{-1}\right\| \leq \frac{1}{d_{\min }(z)-1},
$$

and with probability 1 , this inequality is an equality. Also with probability 1 , the numerical range $W(A)$ is

$$
W(A)=\operatorname{conv}\left(\Omega_{\mathrm{III}}\right) .
$$

Proof. Suppose a bounded inverse $(z-A)^{-1}$ exists for some $z$. As was just noted, one can show by induction starting at the upper left corner that $(z-A)^{-1}$ is upper triangular too and that its entries are given by the same formula (3.1) as for finite-dimensional matrices.

For the first assertion of the theorem, consider $z \in \Omega_{\mathrm{I}}$, i.e., $d_{\max }(z)<1$. If $z \notin \Lambda(A)$, then (3.1) applies, giving us $\left|r_{i j}\right| \geq 1$ for each $i$ and $j$ with $j \geq i$ (in fact with exponential growth). Thus $(z-A)^{-1}$ cannot be bounded after all, and we have a contradiction.

Next, consider $z \in \Omega_{\mathrm{II}} \cup \Omega_{\mathrm{III}}$, i.e., $d_{\min }(z) \leq 1$. Again, if $z \notin \Lambda(A)$, then (3.1) applies, and by parts (II) and (III) of Theorem 1, with probability 1 , there are finite 
sections of $(z-A)^{-1}$ with arbitrarily large norms. Thus with probability $1,(z-A)^{-1}$ is unbounded after all, implying that $z \in \Lambda(A)$. Now this establishes that any fixed $z \in \Omega_{\mathrm{II}} \cup \Omega_{\mathrm{III}}$ is in $\Lambda(A)$ with probability 1 , but the assertion of the theorem is stronger: that with probability 1 all such $z$ are in $\Lambda(A)$. As in the proof of the last theorem, the argument can be expanded to establish this by the use of compactness. As this case is slightly more complicated than that one, we spell out the details. Since $\operatorname{supp}(X)$ is compact, so is $\Omega_{\mathrm{II}} \cup \Omega_{\mathrm{III}}$, and it follows that for any $\epsilon>0$, there exists a finite set of values $z_{j} \in \Omega_{\mathrm{II}} \cup \Omega_{\mathrm{III}}$ such that the union of the $\epsilon$-balls around $\left\{z_{j}\right\}$ covers $\Omega_{\mathrm{II}} \cup \Omega_{\mathrm{III}}$. Our argument implies that with probability 1 , none of the inverses $\left(z_{j}-A\right)^{-1}$ exist as bounded operators. The nonexistence of $\left(z_{j}-A\right)^{-1}$ implies that for $\left|z-z_{j}\right|<\epsilon$, if $(z-A)^{-1}$ exists, then $\left\|(z-A)^{-1}\right\|>\epsilon^{-1}$. Thus we see that for any $\epsilon>0,\left\|(z-A)^{-1}\right\|>\epsilon^{-1}$ uniformly for all $z \in \Omega_{\mathrm{II}} \cup \Omega_{\mathrm{III}}$, with probability 1. Since $\epsilon$ is arbitrary, this implies $\Lambda(A) \supseteq \Omega_{\mathrm{II}} \cup \Omega_{\mathrm{III}}$ with probability 1 .

Now consider $z \in \Omega_{\mathrm{IV}}$, i.e., $d_{\min }(z)>1$. In this case we can use (3.1) to construct an infinite matrix $(z-A)^{-1}$. Regardless of what numbers $x_{j} \in \operatorname{supp}(X)$ appear on the diagonal of $A,(4.7)$ implies that the entries of the matrix decrease exponentially away from the diagonal, and thus it is indeed a bounded inverse of $z-A$. The bound (5.2) can be verified by the same geometric series argument as in the proof of Theorem 1; a weak rather than strict inequality now appears because the series is infinite. Since arbitrarily long stretches of entries arbitrarily close to some $x \in$ $\operatorname{supp}(X)$ with $|z-x|=d_{\min }(z)$ must almost surely appear on the diagonal of $A$, the inequality is almost surely an equality.

Finally, the assertion about $W(A)$ follows essentially as in Theorem 1.

6. Localized and delocalized resolvents. To physicists interested in random matrices related to the Hatano-Nelson example, a crucial phenomenon is that of a delocalization transition. In all previous articles on the subject that we know of, this phenomenon has been approached via the eigenvectors of random matrices of periodic structure, i.e., with $a_{N 1} \neq 0$. It is found that when an eigenvalue $\lambda$ is real, the corresponding eigenvector is exponentially localized in the sense that for some $j_{0}$, its entries decay exponentially with $\left|j-j_{0}\right|$; this is a nonhermitian generalization of the phenomenon of Anderson localization [1]. As one moves along the curve of eigenvalues or changes a parameter so that $\lambda$ becomes complex, on the other hand, the exponential decay is lost and the eigenvectors become global. Questions of localization and delocalization of eigenvectors have been a central topic in condensed matter physics for many years, because they are related to quantum mechanical phenomena such as transparency to light and conductivity to electricity. Hatano, Nelson, and Shnerb have proposed a number of possible physical implications of the delocalization transition for their matrices $[26,27,39]$.

For non-periodic matrices, with $a_{N 1}=0$, the eigenvalues are trivial, and no one has suggested that the eigenvectors corresponding to eigenvalues inside the bubble are physically meaningful. (They are "gauge-transformed Anderson modes," localized at one end, and their exponential growth with the vector index implies that the corresponding eigenvalues are exponentially ill-conditioned; we shall say more about this at the end of the next section.) However, the delocalization phenomenon can still be seen in the resolvent matrix $(z-A)^{-1}$. Mathematically speaking, if a linear system generated by a matrix $A$ is driven at a complex frequency $z$,

$$
\frac{d \phi}{d t}=A \phi+e^{z t} v
$$


for some vector $v$, the solution is

$$
\phi(t)=e^{z t} u, \quad u=(z-A)^{-1} v .
$$

In particular, if we take $v$ to be the discrete delta function $e_{j}$, then $u$ is the $j$ th column of $(z-A)^{-1}$. Thus each column of $(z-A)^{-1}$ represents the response of the system to spatially localized input at frequency $z$, and it is natural to regard $(z-A)^{-1}$ as localized if the entries of these columns decay exponentially with distance from the diagonal, delocalized if they do not.

Our Lemma 1 implies that according to this criterion, $(z-A)^{-1}$ has the following properties as $N \rightarrow \infty$ for $z$ in each of the four regions:

$$
\begin{aligned}
\Omega_{\mathrm{I}} & : \text { delocalized (surely), } \\
\Omega_{\mathrm{II}} & \text { delocalized almost surely, } \\
\Omega_{\mathrm{III}} & \text { localized almost surely, } \\
\Omega_{\mathrm{IV}} & \text { localized (surely). }
\end{aligned}
$$

Figure 6 confirms these predictions for a random matrix with $N=400$. For larger $N$, of course, the distinction between localized and delocalized cases would grow steadily more pronounced. Note that as $z$ approaches the bubble from outside, though the resolvent remains localized, the "correlation lengths" become larger, a familiar phenomenon near critical points in condensed matter.

7. Random bidiagonal periodic matrices. We have considered nonperiodic bidiagonal matrices and shown that they exhibit a Hatano-Nelson bubble and an associated localization-delocalization effect, provided that one looks at pseudospectra and resolvents rather than eigenvalues and eigenvectors. If one looks at eigenvalues and eigenvectors for these matrices, one sees little.

As mentioned in the last section, the usual approach in the literature has been to investigate eigenvalues and eigenvectors, but to do this for matrices of periodic structure, in which the pattern of the superdiagonal entry is continued in the bottomleft corner of the matrix by setting $a_{N 1}=1$. Some authors call a matrix of this structure "periodic bidiagonal". We might also continue the usage of Section 5 and call it "stochastic circulant". In this periodic case it is found that eigenvalues appear that lie along the "bubble" boundary between regions $\Omega_{\mathrm{II}}$ and $\Omega_{\mathrm{III}}$. Goldsheid and Khoruzhenko [24] and Feinberg and Zee [18] have made this precise, and the details of their mathematical arguments are close to ours.

An upper bidiagonal matrix and its periodic variant are closely related, differing only in the $(N, 1)$ position. Since the difference there in our case is 1 , one might think that one problem cannot be regarded as a perturbation of the other that is small in norm. In fact, however, one can interpret a great deal in the light of small perturbations. If $d>1$ is arbitrary, then

$$
D\left(\begin{array}{ccccc}
x_{1} & 1 & & & \\
& x_{2} & 1 & & \\
& & \ddots & \ddots & \\
& & & x_{N-1} & 1 \\
1 & & & & x_{N}
\end{array}\right) D^{-1}=\left(\begin{array}{ccccc}
x_{1} & d & & & \\
& x_{2} & d & & \\
& & \ddots & \ddots & \\
& & & x_{N-1} & d \\
& & & & x_{N}
\end{array}\right)
$$



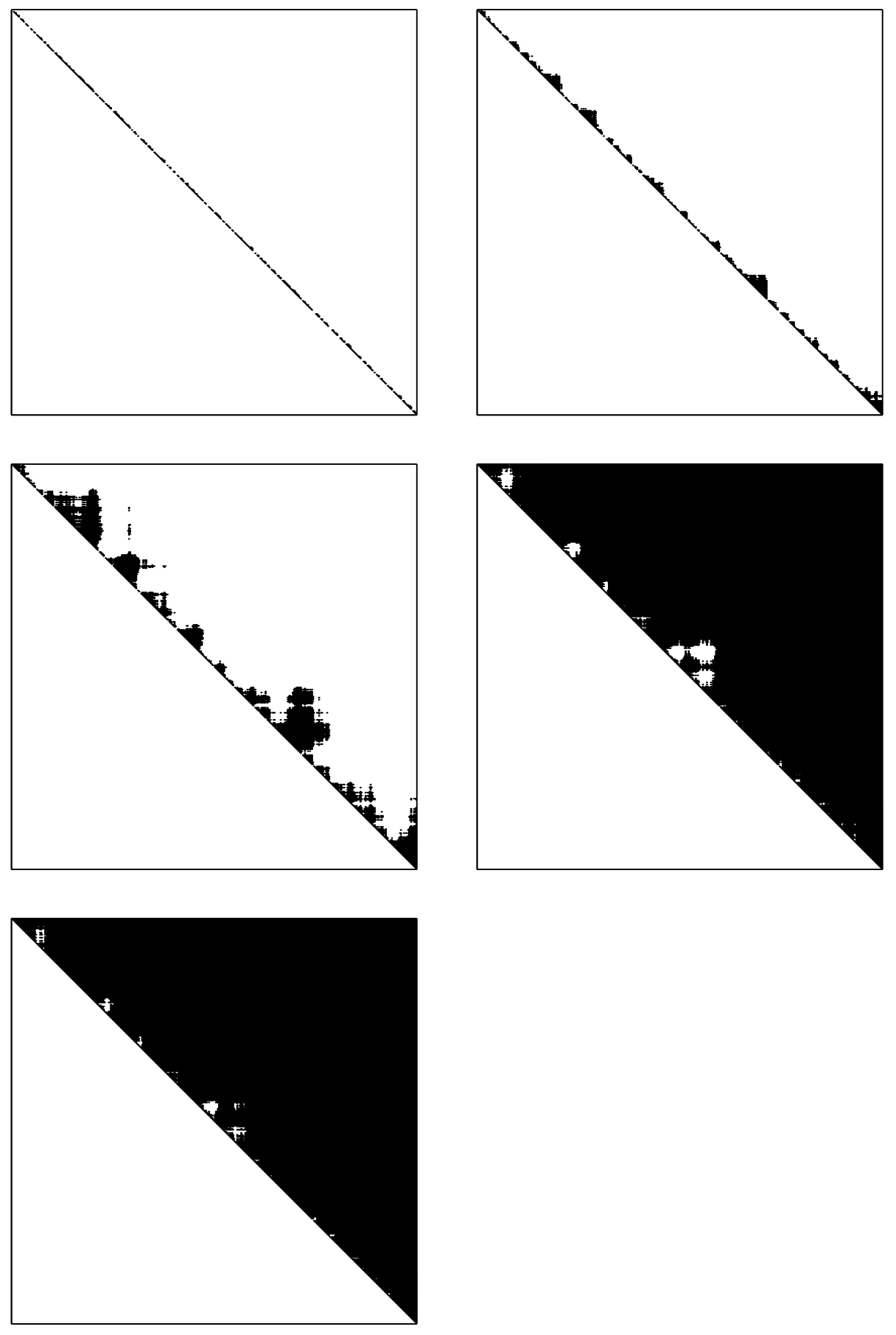

FIG. 6. Localization and delocalization as seen in the resolvents $(z-A)^{-1}$ for a fixed choice of $A \in \operatorname{bidiag}_{N}\{[-2,2], 1\}, N=400$, for the same five values of $z$ as in Figure 3 . A dot is printed at each position of the matrix with $\left|r_{i j}\right|>0.5$. For $z$ outside the bubble (first three plots), the resolvent is concentrated on the diagonal, while for $z$ inside the bubble (last two plots), it is not concentrated. As $z$ approaches the bubble, local exceptions of larger and larger scale appear. 
where $D=\operatorname{diag}\left(d, d^{2}, \ldots, d^{N}\right)$. Mathematicians call this a diagonal similarity transformation; physicists call it a gauge transformation. For large $N$, the corner entry $d^{-N}$ is exponentially small, so the latter matrix is an exponentially small perturbation of a bidiagonal matrix (1.2) with the eigenvalues $x_{1}, \ldots, x_{N}$ and a slightly enlarged diagonal.

Can this exponentially small perturbation change the eigenvalues very much? The following theorem shows that the answer is resoundingly no for some of the eigenvalues, yes for others (those with $d^{-1}<d_{\text {mean }}\left(x_{j}\right)<1$ ). Though not stated in exactly this form before, the statement in the theorem about convergence of the eigenvalues to the "bubble" plus the "wings" is essentially contained in the papers of Brézin, Feinberg, Goldsheid, Khoruzhenko, and Zee. We define these two sets precisely, for general $X$, as follows:

$$
\begin{aligned}
S_{\text {bubble }} & \equiv\left\{z \in \mathbf{C}: d_{\text {mean }}(z)=1\right\} \\
S_{\text {wings }} & \equiv\left\{z \in \operatorname{supp}(X): d_{\text {mean }}(z)>1\right\} .
\end{aligned}
$$

The set $S_{\text {wings }}$ may contain curves and regions with interior, but it cannot contain isolated points, for if an isolated point $z_{0}$ belongs to $\operatorname{supp}(X)$, then the measure $\mu\left(z_{0}\right)$ must be positive, implying $d_{\text {mean }}\left(z_{0}\right)=0$. In the unbounded region exterior to $\operatorname{supp}(X), S_{\text {bubble }}$ can consist only of closed curves with $d_{\text {mean }}(z)>1$ on one side and $d_{\text {mean }}(z)<1$ on the other, for it is a level set of the harmonic function $E(\log |z-X|)$, which satisfies the maximum principle, yet is not constant since it increases to $\infty$ as $z \rightarrow \infty$. Within and interior to $\operatorname{supp}(X), S_{\text {bubble }}$ can be more complicated. For example, if $X$ is the uniform distribution on the unit circle, then $S_{\text {bubble }}$ is the closed unit disk.

TheOREM 3 (random bidiagonal periodic matrices). Let $A$ be an $N \times N$ matrix ("stochastic bidiagonal circulant matrix") of the form (1.2), except with $a_{N 1}=1$, and let $z \in \mathbf{C}$ be given. If $z \in \Omega_{\mathrm{I}}$, then $\left\|(z-A)^{-1}\right\| \leq 1 /\left(1-d_{\max }(z)\right)$, and if $z \in \Omega_{\mathrm{IV}}$, then $\left\|(z-A)^{-1}\right\| \leq 1 /\left(d_{\min }(z)-1\right)$. If $z \in \Omega_{\mathrm{II}} \cup \Omega_{\mathrm{III}}$, then $\left\|(z-A)^{-1}\right\| \rightarrow \infty$ a.s. as $N \rightarrow \infty$, and if in addition $z \notin S_{\text {bubble }} \cup S_{\text {wings }}$, then $\left\|(z-A)^{-1}\right\|^{1 / N} \rightarrow 1$ a.s. as $N \rightarrow \infty$. Moreover,

$$
W(A) \rightarrow \operatorname{conv}\left(\Omega_{\mathrm{III}}\right)
$$

almost surely as $N \rightarrow \infty$, and, provided that $S_{\text {bubble }}$ consists only of curves disjoint from $\operatorname{supp}(X)$ except at isolated points,

$$
\Lambda(A) \rightarrow S_{\text {bubble }} \cup S_{\text {wings }}
$$

almost surely as $N \rightarrow \infty$.

Proof. First, consider $z \in \Omega_{\mathrm{I}}$, i.e., $d_{\max }(z)<1$. Let $S$ denote the circulant shift matrix with $s_{i j}=1$ for $j \equiv(i+1)(\bmod N), 0$ otherwise. Then we have

$$
z-A=D-S
$$

where $D$ is the diagonal matrix with entries $z-x_{j}$. Thus we can regard $z-A$ as a perturbation of norm $\|D\| \leq d_{\max }(z)$ of $-S$. It follows that $z-A$ has an inverse, and it satisfies $\left\|(z-A)^{-1}\right\| \leq 1 /\left(1-d_{\max }(z)\right)$.

Next, suppose $z \in \bar{\Omega}_{\mathrm{II}} \cup \Omega_{\mathrm{III}}$. An argument to establish $\left\|(z-A)^{-1}\right\| \rightarrow \infty$ a.s. can be made that is essentially the same as in the proof of the corresponding part of 
Theorem 4 in the next section; we give details there. Conversely, we must show that if $z \notin S_{\text {bubble }} \cup S_{\text {wings }}$, the growth is not exponential. We return to this at the end of the proof.

If $z \in \Omega_{\mathrm{IV}}$, again we make use of (7.4), but now it is $\left\|D^{-1}\right\|$ rather than $\|D\|$ that is small. Since $d_{\min }(z)>1$, we now have $\left\|D^{-1}\right\| \leq 1 / d_{\min }(z)<1$, hence $\left\|S D^{-1}\right\|<1$ also, giving a convergent series representation

$$
(z-A)^{-1}=(D-S)^{-1}=D^{-1}+D^{-1}\left(S D^{-1}\right)+D^{-1}\left(S D^{-1}\right)^{2}+\cdots
$$

and the required bound.

The assertions concerning $W(A)$ are corollaries of the facts that $\left\|(z-A)^{-1}\right\| \rightarrow \infty$ a.s. as $N \rightarrow \infty$ for $z \in \Omega_{\mathrm{II}} \cup \Omega_{\mathrm{III}}$ and that $\left\|(z-A)^{-1}\right\| \leq 1 /\left(d_{\min }(z)-1\right)$ for $z$ outside this region, as in the proofs of the last two theorems.

The claim (7.3) regarding the spectrum is essentially as in the papers by Goldsheid and Khoruzhenko and Feinberg and Zee. A proof can be based on the characteristic equation of $A$, which is easily seen to be

$$
p(z)=\prod_{j=1}^{N}\left(x_{j}-z\right)=1 .
$$

We sketch the argument without giving details, and without repeating in each clause the qualification "almost surely as $N \rightarrow \infty$." By methods akin to those of earlier proofs in this paper it can be seen that if $d_{\text {mean }}(z)<1$, i.e., for $z \in \Omega_{\mathrm{I}} \cup \Omega_{\mathrm{II}},(7.6)$ cannot hold: $p(z)$ is exponentially smaller in magnitude than 1 . For $d_{\text {mean }}(z)>1$, we similarly have that $p(z)$ is exponentially larger than 1 , except that if $z \in \operatorname{supp}(X)$, then equality may be achieved by taking $z$ exceptionally close to some $x_{j}$. (A physicist might say that an eigenvalue is pinned to an impurity.) Thus any limit points of $\Lambda(A)$ as $N \rightarrow \infty$ must be confined to $S_{\text {bubble }} \cup S_{\text {wings }}$. Conversely, we must show that every $z \in S_{\text {bubble }} \cup S_{\text {wings }}$ is such a limit point. This can be done with the aid of the principle of the argument of complex analysis, which asserts that if $p(z)$ maps a closed loop $\Gamma$ onto a curve that winds $\nu$ times counterclockwise around the point 1 , then the equation $p(z)=1$ has $\nu$ roots interior to $\Gamma$, counted with multiplicity. For $z \in S_{\text {wings }}$, since $d_{\text {mean }}(z)>1$, we can construct arbitrarily small loops $\Gamma$ near $z$ that enclose one or more points $x_{j}$ but satisfy $|p(\zeta)|>1$ for all $\zeta \in \Gamma$; by the argument principle, there are as many eigenvalues $\lambda$ inside such a loop as there are points $x_{j}$. Note that this shows not only that the eigenvalues cluster on $\operatorname{supp}(X)$ for $d_{\text {mean }}(z)>1$, but that the densities of the eigenvalues match those of the samples from $\operatorname{supp}(X)$.

For $z \notin \operatorname{supp}(X)$ lying on an arc of $S_{\text {bubble }}$, as noted above, we have $d_{\text {mean }}(\zeta)>1$ on one side of the arc and $d_{\text {mean }}(\zeta)<1$ on the other. It follows that the same holds for $|p(\zeta)|$, implying that a small loop $\Gamma$ can be constructed near $z$ such that $p(\Gamma)$ winds counterclockwise around the outside of the unit disk a number of times, cuts inside without passing through the point 1 , winds around inside, then cuts back out again. Such a curve has positive winding number about 1 , and thus again there are eigenvalues near $z$.

Finally we return to the proof that for $z \notin S_{\text {bubble }} \cup S_{\text {wings }}$, though $\left\|(z-A)^{-1}\right\|$ may diverge to $\infty$ as $N \rightarrow \infty$, the growth is almost surely not exponential. For this, as in the proof of Theorem 1, it is convenient to use an explicit formula for $(z-A)^{-1}$. 
From Cramer's Rule we find, in analogy to (3.1),

$$
r_{i j}= \begin{cases}\prod_{k<i \text { or } k>j}\left(z-x_{k}\right) /\left(-1+\prod_{\text {all } k}\left(z-x_{k}\right)\right), & i \leq j, \\ \prod_{k<i \text { and } k>j}\left(z-x_{k}\right) /\left(-1+\prod_{\text {all } k}\left(z-x_{k}\right)\right), & i>j .\end{cases}
$$

For $z \notin S_{\text {bubble }} \cup S_{\text {wings }}$, as argued above, $\prod_{\text {all } k}$ is almost surely exponentially smaller (inside the bubble) or larger than 1 (outside). In the former case the denominators in (7.7) are $\approx-1$ and the numerators are $O(1)$, so $r_{i j}$ is $O(1)$. In the latter case the denominators are exponentially large and the numerators are exponentially large too, but as there are fewer factors $\left(z-x_{k}\right)$ in the numerators, the ratio is again $O(1)$. Thus in either case $r_{i j}$ is $O(1)$, and a complete proof that this gives the required behavior a.s. as $N \rightarrow \infty$ can be based on Lemma 2, as in the proof of Theorem 1 .

The papers by Feinberg and Zee and Goldsheid and Khoruzhenko carry arguments like these further to obtain estimates not just of where the eigenvalues lie, but of their density. These are essentially arguments of potential theory. If an eigenvalue $\lambda$ of $A$ is thought of as a point charge with potential $N^{-1} \log |z-\lambda|$, then in the limit $N \rightarrow \infty, S_{\text {bubble }}$ is an equipotential surface of potential zero, and the eigenvalues lie along it in an equilibrium configuration subject to the external field applied by the other eigenvalues, if any, pinned to the values $x_{j}$ in $S_{\text {wings }}$.

In this article we have hardly mentioned condition numbers of eigenvalues, which measure sensitivity of individual eigenvalues to perturbations [12], but they are implicit in any discussion of pseudospectra, and Davies has investigated eigenvalue condition numbers for his random tridiagonal matrices [11]. If $x_{j}$ is a simple eigenvalue of a bidiagonal matrix $A$, the condition number of $x_{j}$ is $\kappa\left(x_{j}\right)=1 /\left|w^{*} v\right|$, where $w^{*}$ and $v$ are normalized left and right eigenvectors of $A$ associated with $x_{j}$, respectively. If $x_{j}$ is in $S_{\text {wings }}$, both $w^{*}$ and $v$ are localized and $\kappa\left(x_{j}\right)=O(1)$, whereas if $d_{\text {mean }}\left(x_{j}\right)<1, \kappa\left(x_{j}\right)$ is of order $d_{\text {mean }}\left(x_{j}\right)^{-N}$. This huge condition number gives another perspective on how it is possible that introducing a corner entry can move all the eigenvalues with $d_{\text {mean }}\left(x_{j}\right)<1$ out to the bubble. For $x_{j} \in S_{\text {wings }}$, the condition number can also be used to give an alternative proof that introducing the corner entry has negligible effect on this eigenvalue. At first one might think, since $a_{N 1}=1$ and $\kappa\left(x_{j}\right)=O(1)$, that introducing the corner entry could move the eigenvalue by a distance $O(1)$, but with the help of $(7.1)$ we see that nothing like that can happen. If $d$ is chosen slightly less than $d_{\text {mean }}\left(x_{j}\right)$, the bidiagonal part of the right-hand matrix of (7.1) still has localized left and right eigenvectors and $\kappa\left(x_{j}\right)=O(1)$, but it is only a distance $d^{-N} \approx d_{\text {mean }}\left(x_{j}\right)^{-N}$ from the periodic matrix. Thus introducing the corner entry in fact perturbs the eigenvalue $x_{j}$ at most on the order of $d_{\text {mean }}\left(x_{j}\right)^{-N}$.

8. Random bidiagonal doubly infinite matrices. Finally we turn to the doubly infinite or Laurent case. Questions of spectra have been considered for a class of tridiagonal doubly infinite random operators by Davies [11], and our arguments are close to his. Some results on such spectra are also mentioned by Goldsheid and Khoruzhenko [24].

It is well known that whereas the spectrum of a Toeplitz operator is a certain curve in the complex plane (the image of the unit circle under the function known as the symbol of the operator) together with the points it encloses $[6,22,57]$, the spectrum 
of the corresponding Laurent operator is just the curve itself $[6,14,57]$. The simplest example is the shift operator $S$ with $s_{i, i+1}=1$ for each $i$ but $s_{i j}=0$ otherwise, which has spectrum equal to the closed unit disk if it is singly infinite, but just the unit circle if it is doubly infinite. Part of the explanation is that in the doubly infinite case, the inverse of an upper triangular operator need not be upper triangular. Indeed, for this example the inverse is the lower triangular reverse-shift operator defined by $\left(S^{-1}\right)_{i+1, i}=1$.

For us, the shift operator is more than just an example, for as in the proof of Theorem 3, our infinite matrix $A$ can be regarded as a diagonal perturbation of the doubly infinite shift operator $S$, which is a normal operator $\left(S S^{*}=S^{*} S\right)$. Therefore, introducing the diagonal entries can only perturb the spectrum - the unit circle-by at $\operatorname{most}_{\sup _{j}}\left|x_{j}\right|[32]$.

THEOREM 4 (random bidiagonal doubly infinite matrices). Let $A$ be a doubly infinite matrix ("stochastic Laurent operator") of the form (1.2). Then $\Lambda(A) \subseteq \Omega_{\mathrm{II}} \cup$ $\Omega_{\mathrm{III}}$, and with probability 1 ,

$$
\Lambda(A)=\Omega_{\mathrm{II}} \cup \Omega_{\mathrm{III}}
$$

For $z \in \Omega_{\mathrm{I}}$ we have $\left\|(z-A)^{-1}\right\| \leq 1 /\left(1-d_{\max }(z)\right)$, for $z \in \Omega_{\mathrm{IV}}$ we have $\left\|(z-A)^{-1}\right\| \leq$ $1 /\left(d_{\min }(z)-1\right)$, and with probability 1 , both of these inequalities are equalities. Also with probability $1, W(A)=\operatorname{conv}\left(\Omega_{\mathrm{III}}\right)$.

Proof. First, consider $z \in \Omega_{\mathrm{I}}$, i.e., $d_{\max }(z)<1$. As in the proof of Theorem 3 , we make use of equation (7.4), where now $S$ denotes the doubly infinite shift operator. As in the that proof, since $z-A$ is a perturbation of $-S$ of norm $\leq d_{\max }(z)$, it has an inverse satisfying $\left\|(z-A)^{-1}\right\| \leq 1 /\left(1-d_{\max }(z)\right)$. Since arbitrarily long stretches of entries arbitrarily close to some $x \in \operatorname{supp}(X)$ with $|z-x|=d_{\max }(z)$ must almost surely appear on the diagonal of $A$, the inequality is almost surely an equality.

Next, suppose $z \in \Omega_{\mathrm{II}} \cup \Omega_{\mathrm{III}}$. Suppose the diagonal entries of $A$ happen to consist of a fixed finite set of numbers $x_{1}, \ldots, x_{J} \in \operatorname{supp}(X)$ repeating periodically. Then by standard theory of spectra of block Laurent operators [23,36], it is known that the spectrum of $A$ is $\left\{\lambda \in \mathbf{C}:\left|\left(\lambda-x_{1}\right) \cdots\left(\lambda-x_{J}\right)\right|=1\right\}$, and that for each $\lambda \in \Lambda(A)$ there is a corresponding eigenvector in $\ell_{\infty}(\mathbf{Z})$, finite sections of which give arbitrarily good approximate eigenvectors in $\ell_{2}(\mathbf{Z})$. Now since $d_{\min }(z) \leq 1 \leq d_{\max }(z)$, we can pick such a set $x_{1}, \ldots, x_{J}$ whose geometric mean distance to $z$ is as close as we wish to 1 , implying that there are values $\lambda \in \Lambda(A)$ as close as we wish to $z$. Since $A$ is an infinite matrix, arbitrarily large finite sections must almost surely appear that come arbitrarily close to this particular periodic structure. Therefore the random matrix must also have arbitrarily good approximate eigenvectors in $\ell_{2}(\mathbf{Z})$.

If $z \in \Omega_{\mathrm{IV}}$, we repeat the argument of Theorem 2 or Theorem 3: construct an upper triangular operator $(z-A)^{-1}$ by $(3.1)$ or $(7.5)$ and verify that it is a bounded inverse with the necessary properties.

The assertions concerning $\Lambda(A)$ and $W(A)$ follow as in the previous theorems. 口

In the proof just given we have noted that for $z \in \Omega_{\mathrm{IV}},(z-A)^{-1}$ is upper triangular, whereas for $d_{\max }(z)<1$, it is lower triangular. This difference in structure from one region to another is a familiar phenomenon in the theory of Toeplitz operators, where the crucial quantity is the winding number about a point $z$ in the complex plane of the image of the unit circle under the symbol $[6,14]$. Here, because of randomness, the curve broadens to a belt of finite thickness. Actually, our hypotheses 
do not require $X$ to be random. If it is constant, we just have bidiagonal Toeplitz matrices, and Theorems 2 and 4 reduce to the familiar result that $\Lambda(A)$ is a closed disk and a circle, respectively.

9. Summary and discussion. In this article we have not discussed the HatanoNelson model explicitly, but those familiar with this area will recognize that it has been a motivating force throughout. The Hatano-Nelson model is a tridiagonal analogue of (1.2) in which both the sub- and super-diagonal entries are nonzero, but distinct. Their analogous notation to that of (1.1) would be

$$
\operatorname{tridiag}_{N}\left\{\frac{t}{2} e^{-g}, X, \frac{t}{2} e^{+g}\right\}
$$

where $t$ and $g \geq 0$ are real parameters. The papers $[17,18,24,26,27,39]$ all deal with the eigenvalues of such matrices in the periodic case $a_{N 1}=e^{+g}$, and there are many more we have not cited.

We developed the observations of this paper originally for Hatano-Nelson matrices. In particular, in the nonperiodic tridiagonal case one has an exponentially growing resolvent norm inside the bubble and a subexponentially growing norm in a bounded region outside. We have confined our treatment here to the bidiagonal case because it captures the essence of the matter and yet is essentially simple. Table 1 summarizes the facts about these matrices established in our four theorems. It would probably not be a big step to work out analogues of most of these results for tridiagonal matrices. Much of the work has already been done in the papers of Brézin, Feinberg, and Zee [7,17,18], Goldsheid and Khoruzhenko [24], and Davies [11].

Speaking as mathematicians, we propose that it would be interesting to see how far the theorems summarized in Table 1 can be generalized to more general "stochastic Toeplitz and circulant/Laurent matrices and operators."

Speaking as physicists, we note that in a wide range of applications involving strongly non-normal matrices and operators, as listed in the introduction, pseudospectra have been found to have greater physical significance than spectra. It will be interesting to see what happens when physical systems are eventually constructed in the laboratory that are governed by matrices of the Hatano-Nelson type. We expect that for non-periodic systems of this kind, it may once again be the pseudospectra that match the experiments.

Acknowledgments. We have benefitted from conversations with A. Böttcher, E. B. Davies, A. Etheridge, B. Khoruzhenko, and G. Strang. We are especially grateful to D. Loghin for early development of some of these results.

\section{REFERENCES}

[1] P. Anderson, Absence of diffusion in certain random lattices, Phys. Rev., 109 (1958), pp. $1492-1505$.

[2] J. S. Baggett, Pseudospectra of an operator of Hille and Phillips, Res. Rep. 94-15, Interdisc. Proj. Ctr. Supercomp., Swiss Federal Institute of Technology, Zurich, 1994.

[3] M. V. BerRy AND J. P. KeAting, The Riemann zeros and eigenvalue asymptotics, SiAM Review, 41 (1999), pp. 236-266.

[4] D. BORBA, ET AL., The pseudospectrum of the resistive magnetohydrodynamics operator: resolving the resistive Alfvén paradox, Phys. Plasmas, 1 (1994), pp. 3151-3160.

[5] A. BötTcher, Pseudospectra and singular values of large convolution operators, J. Int. Eqs. Appl., 6 (1994), pp. 267-301.

[6] A. Böttcher And B. Silbermann, Introduction to Large Truncated Toeplitz Matrices, Springer, New York, 1998. 
[7] E. BRÉzin AND A. ZeE, Non-Hermitean delocalization: multiple scattering and bounds, Nuc. Phys. B, 509 (1998), pp. 599-614.

[8] F. Chattin-Chatelin and V. Frayssé, Lectures on Finite Precision Computations, SiAM, Philadelphia, 1996.

[9] E. B. Davies, Pseudospectra, the harmonic oscillator and complex resonances, Proc. Roy. Soc. Lond. A, 455 (1999), pp. 585-599.

[10] E. B. DAviEs, Pseudospectra of differential operators, J. Operator Theory, to appear.

[11] E. B. DAVIES, Spectral properties of random non-self-adjoint matrices and operators, preprint, Los Alamos e-Print archives xxx.lanl.gov, math/0002159.

[12] J. W. Demmel, Applied Numerical Linear Algebra, SIAM, Philadelphia, 1997.

[13] J. L. M. van Dorsselaer, J. F. B. M. KraAijevanger and M. N. SpIJKer, Linear stability analysis in the numerical solution of initial value problems, Acta Numerica, 1993 (1993), pp. 199-237.

[14] R. G. Douglas, Banach Algebra Techniques in Operator Theory, Academic Press, New York, 1972.

[15] A. Edelman, Eigenvalues and Condition Numbers of Random Matrices, PhD thesis, MIT, 1989.

[16] M. Embree and L. N. Trefethen, Pseudospectra of random triangular matrices, in preparation.

[17] J. Feinberg And A. Zee, Non-Hermitian localization and delocalization, Phys. Rev. E, 59 (1999), pp. 6433-6443 (Los Alamos e-Print archives xxx.lanl.gov, cond-mat/ 9706218).

[18] J. Feinberg and A. Zee, Spectral curves of non-hermitian hamiltonians, Nucl. Phys. B, 552 (1999), pp. 599-623 (Los Alamos e-Print archives xxx.lanl.gov, cond-mat/9710040).

[19] J. Ginibre, Statistical ensembles of complex, quaternion, and real matrices, J. Math. Phys., 6 (1965), pp. 440-449.

[20] I. C. Goberg And M. G. KRein, Introduction to the Theory of Linear Nonselfadjoint Operators, Amer. Math. Soc., Providence, RI, 1969.

[21] S. K. Godunov, O. P. Kiriluuk and V. I. Kostin, Spectral portraits of matrices, Tech. Rep. 3, Inst. of Math., Acad. Sci. USSR, Novosibirsk, 1990.

[22] I. GoHBERG, On the application of the theory of normed rings to singular integral equations, Uspekhi Mat. Nauk, 7 (1952), pp. 149-156 (Russian).

[23] I. C. Gohberg And I. A. Fel'Dman, Convolution Equations and Projection Methods for Their Solution, Amer. Math. Soc., Providence, RI, 1974.

[24] I. Ya. GOLDSheid AND B. A. KHORUZHenKo, Distribution of eigenvalues in non-Hermitian Anderson models, Phys. Rev. Lett., 80 (1998), pp. 2897-2900 (a different version of this paper appears in Los Alamos e-Print archives xxx.lanl.gov, cond-mat/9707230).

[25] G. R. Grimmett and D. R. Stirzaker, Probability and Random Processes, 2nd ed., Oxford U. Press, Oxford, 1992.

[26] N. Hatano and D. R. Nelson, Localization transitions in non-hermitian quantum mechanics, Phys. Rev. Lett., 77 (1996), pp. 570-473.

[27] N. Hatano and D. R. Nelson, Vortex pinning and non-Hermitian quantum mechanics, Phys. Rev. B, 56 (1997), pp. 8651-8673 (Los Alamos e-Print archives xxx.lanl.gov, condmat/9705290).

[28] D. S. Henningson and P. J. Schmid, Stability and Transition in Shear Flows, Springer-Verlag, Berlin, 2000.

[29] D. J. Higham and L. N. Trefethen, Stiffness of ODEs, BIT, 33 (1993), pp. 285-303.

[30] D. HinRichsen And B. Kelb, Spectral value sets: a graphical tool for robustness analysis, Systems and Control Letters, 21 (1993), pp. 127-136.

[31] G. F. Jónsson and L. N. Trefethen, A numerical analyst looks at the "cutoff phenomenon" in card shuffling and other Markov chains, in D. F. Griffiths, D. J. Higham and G. A. Watson, eds., Numerical Analysis 1997, Longman Scientific and Technical, Harlow, Essex, UK, 1998 , pp. $150-178$.

[32] T. Kato, Perturbation Theory for Linear Operators, 2nd ed., Springer-Verlag, New York, 1976.

[33] N. M. Katz and P. Sarnak, Random Matrices, Frobenius Eigenvalues, and Monodromy, AMS, Providence, RI, 1999.

[34] V. I. KRYlov, Approximate Calculation of Integrals, Macmillan, New York, 1962.

[35] H. J. Landau, On Szegö's eigenvalue distribution theory and non-Hermitian kernels, J. d'Analyse Math., 28 (1975), pp. 335-357.

[36] L. E. LERER, On the asymptotic distribution of the spectra of finite truncations of Wiener-Hopf operators, Dokl. Akad. Nauk SSSR, 207 (1972), pp. 1651-1655.

[37] M. L. Menta, Random Matrices and the Statistical Theory of Energy Levels, Academic Press, New York, 1967. 
[38] R. J. Mutrhead, Aspects of Multivariate Statistical Theory, Wiley, New York, 1982.

[39] D. R. Nelson and N. M. Shnerb, Non-hermitian localization and population biology, Phys. Rev. E., 58 (1998), pp. 1383-1403 (Los Alamos e-Print archives xxx.lanl.gov, condmat/9708071).

[40] R. Plato, Resolvent estimates for Abel integral operators and the regularization of associated first kind integral equations, J. Int. Eqs. Appl., 9 (1997), pp. 253-278.

[41] S. C. REDdy, Pseudospectra of Wiener-Hopf integral operators and constant-coefficient differential operators, J. Int. Eqs. Appl., 5 (1993), pp. 369-403.

[42] S. C. Reddy and L. N. Trefethen, Stability of the method of lines, Numer. Math., 62 (1992), pp. $235-267$.

[43] S. C. RedDy ANd L. N. TREFEthen, Pseudospectra of the convection-diffusion operator, SIAM J. Appl. Math., 54 (1994), pp. 1634-1649.

[44] M. Reed And B. Simon, Methods of Modern Mathematical Physics I: Functional Analysis, Academic Press, San Diego, 1980.

[45] L. Reichel and L. N. Trefethen, Eigenvalues and pseudo-eigenvalues of Toeplitz matrices, Lin. Alg. Appl., 162-164 (1992), pp. 153-185.

[46] K.-C. Toh AND L. N. TRefethen, Pseudozeros of polynomials and pseudospectra of companion matrices, Numer. Math., 68 (1994), pp. 403-425.

[47] L. N. Trefethen, Approximation theory and numerical linear algebra, in J. C. Mason and M. G. Cox, eds., Algorithms for Approximation II, Chapman and Hall, London, 1990, pp. $336-360$.

[48] L. N. Trefethen, Pseudospectra of matrices, in D. F. Griffiths and G. A. Watson, eds., Numerical Analysis 1991, Longman Scientific and Technical, Harlow, Essex, UK, 1992, pp. $234-266$.

[49] L. N. Trefethen, Pseudospectra of linear operators, SIAM Review, 39 (1997), pp. 383-406.

[50] L. N. Trefethen, unpublished letter to D. Nelson of Harvard University, 19 November 1997.

[51] L. N. TRefethen, Spectra and pseudospectra: the behavior of non-normal matrices and operators, in M. Ainsworth, J. Levesley and M. Marletta, The Graduate Student's Guide to Numerical Analysis, Springer, Berlin, 1999, pp. 217-250.

[52] L. N. Trefethen, Computation of pseudospectra, Acta Numerica, 8 (1999), pp. 247-295.

[53] L. N. Trefethen, Spectral Methods in MATLAB, SIAM, Philadelphia, 2000.

[54] L. N. Trefethen, A. E. Trefethen, S. C. Reddy and T. A. Driscoll, Hydrodynamic stability without eigenvalues, Science, 261 (1993), pp. 578-584.

[55] J. M. VARAH, On the separation of two matrices, SIAM J. Numer. Anal., 16 (1979), pp. $216-$ 222.

[56] D. Viswanath and L. N. Trefethen, Condition numbers of random triangular matrices, SIAM J. Matrix Anal. Appl., 19 (1998), pp. 564-581.

[57] H. Widom, Toeplitz matrices, in I. I. Hirschman, Jr., ed., Studies in Real and Complex Analysis, Math. Assoc. Amer., Washington, DC, 1965.

[58] E. P. Wigner, Random matrices in physics, SIAM Review, 9 (1967), pp. 1-23.

[59] J. WISHART, The generalized product moment distribution in samples from a normal multivariate population, Biometrika, 20A (1928), pp. 32-43. 
TABLe 1.

Summary of results.

$\begin{array}{cccc}\text { Theorem } 1 & \text { Theorem } 2 & \text { Theorem } 3 & \text { Theorem } 4 \\ \text { "stochastic } & \text { "stochastic } & \text { "stochastic } & \text { "stochastic } \\ \text { bidiagonal } & \text { bidiagonal } & \text { bidiagonal } & \text { bidiagonal } \\ \text { Toeplitz } & \text { Toeplitz } & \text { circulant } & \text { Laurent } \\ \text { matrix" } & \text { operator" } & \text { matrix" } & \text { operator" } \\ \text { non-periodic } & \text { non-periodic } & \text { periodic } & \text { periodic } \\ N<\infty & N=\infty & N<\infty & N=\infty\end{array}$

RESOLVENT NORMS

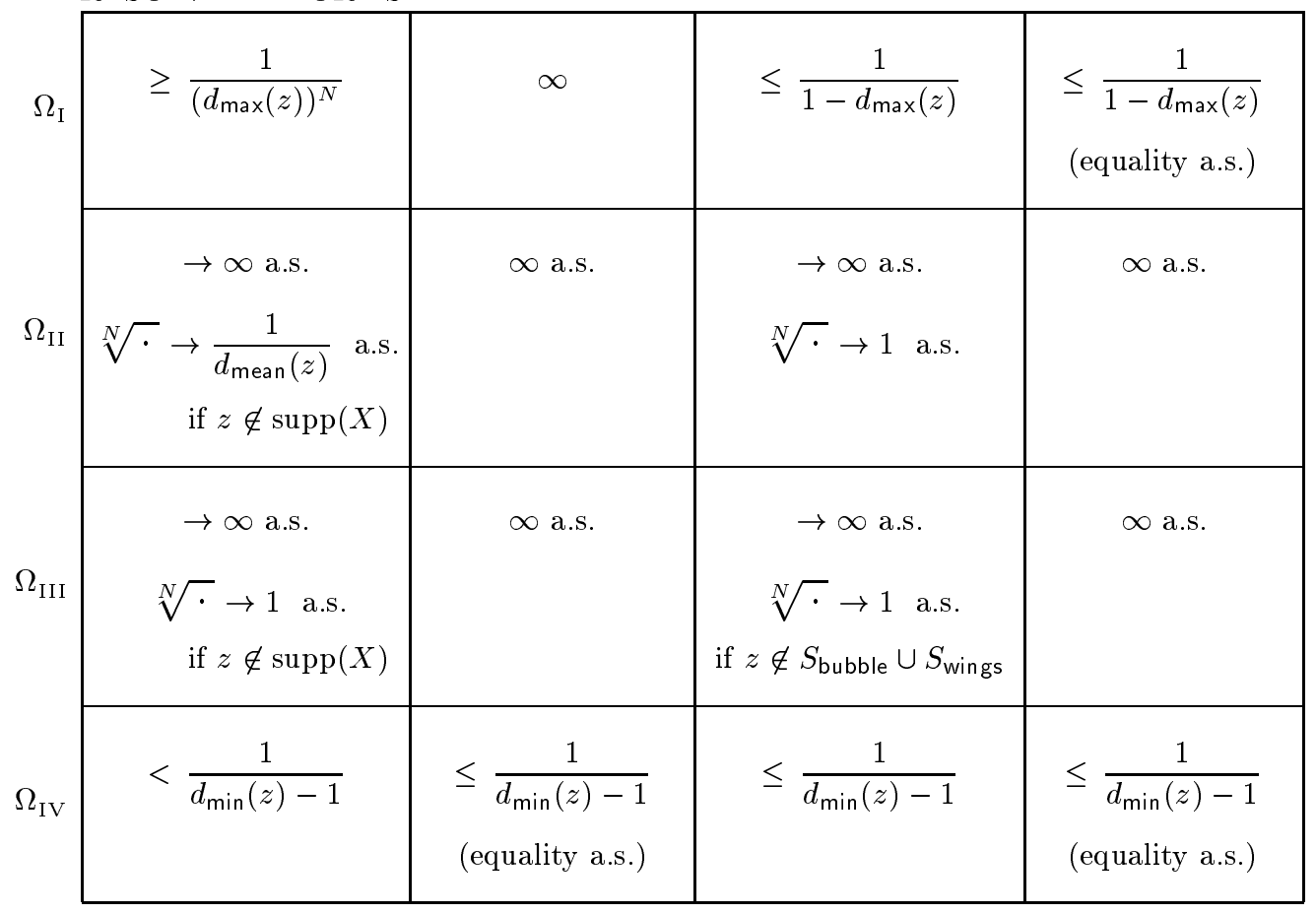

\section{SPECTRUM AND NUMERICAL RANGE}

\begin{tabular}{|c|c|c|c|c|}
\hline$\Lambda(A)$ & $\rightarrow \operatorname{supp}(X)$ a.s. & $\Omega_{\mathrm{I}} \cup \Omega_{\mathrm{II}} \cup \Omega_{\mathrm{III}}$ a.s. & $\rightarrow S_{\text {bubble }} \cup S_{\text {wings }}$ a.s. & $\Omega_{\mathrm{II}} \cup \Omega_{\mathrm{III}}$ a.s. \\
\hline$W(A)$ & $\rightarrow \operatorname{conv}\left(\Omega_{\mathrm{III}}\right)$ a.s. & $\operatorname{conv}\left(\Omega_{\mathrm{III}}\right)$ a.s. & $\rightarrow \operatorname{conv}\left(\Omega_{\mathrm{III}}\right)$ a.s. & $\operatorname{conv}\left(\Omega_{\mathrm{III}}\right)$ a.s. \\
\hline
\end{tabular}

